\title{
TITLE:
}

\section{Mobile PEAR transcription factors integrate positional cues to prime cambial growth}

\section{AUTHORS:}

Shunsuke Miyashima ${ }^{2,3 *}$, Pawel Roszak ${ }^{1,2 *}$, Iris Sevilem ${ }^{2 *}$, Koichi Toyokura ${ }^{1,7 * *}$, Bernhard Blob $^{1 * *}$, Jung-ok Heo ${ }^{1,2}$, Nathan Mellor ${ }^{8}$, Hanna Help-Rinta-Rahko ${ }^{2}$, Sofia Otero ${ }^{1}$, Wouter Smet $^{4,5,6}$, Mark Boekschoten ${ }^{14}$, Guido Hooiveld ${ }^{14}$, Kayo Hashimoto ${ }^{3,9}$, Ondřej Smetana ${ }^{2}$, Riccardo Siligato $^{2}$, Eva-Sophie Wallner ${ }^{12}$, Ari Pekka Mähönen ${ }^{2}$, Yuki Kondo ${ }^{10}$, Charles W Melnyk $^{1,11}$, Thomas Greb ${ }^{12}$, Keiji Nakajima ${ }^{3}$, Rosangela Sozzani ${ }^{13}$, Anthony Bishopp ${ }^{8}$, Bert De Rybel $^{4,5,6 \#}$, Ykä Helariutta ${ }^{1,2}$ \#

\section{AFFILIATIONS:}

1: The Sainsbury Laboratory, University of Cambridge, Cambridge, CB2 1LR, UK

2: Institute of Biotechnology, HiLIFE/Organismal and Evolutionary Biology Research Programme, Faculty of Biological and Environmental Sciences, and Viikki Plant Science Centre, University of Helsinki, FIN-00014, Finland

3: Graduate School of Science and Technology, Nara Institute of Science and Technology, Nara, 6300192 Japan

4: Ghent University, Department of Plant Biotechnology and Bioinformatics, 9052 Ghent, Belgium

5: VIB Center for Plant Systems Biology, 9052 Ghent, Belgium

6: Wageningen University, Laboratory of Biochemistry, $6708 \mathrm{WE}$, Wageningen, The Netherlands

7: Department of Biological Sciences, Graduate School of Science, Osaka University, Toyonaka, Osaka, 560-0043, Japan

8: Centre for Plant Integrative Biology (CPIB) and School of Biosciences, University of Nottingham, Nottingham, LE12 5RD, UK

9: Graduate School of Humanities and Sciences, Nara Women's University, Nara, 630-8506 Japan

10: Department of Biological Sciences, Graduate School of Science, The University of Tokyo, Tokyo, 113-0033, Japan

11: Department of Plant Biology, Swedish University of Agricultural Sciences, 75651 Uppsala, Sweden

12: Centre for Organismal Studies (COS), Heidelberg University, 69120 Heidelberg, Germany

13: Department of Plant and Microbial Biology, North Carolina State University, Raleigh, NC 27695, United States.

14: Nutrition, Metabolism and Genomics Group, Division of Human Nutrition, Wageningen University, 6708 WE Wageningen, The Netherlands

\# Corresponding author. Email: Ykä Helariutta: yrjo.helariutta@slcu.cam.ac.uk; Bert De Rybel: beryb@psb.vib-ugent.be

* These authors contributed equally to this work.

** These authors contributed equally to this work. 
While apical growth in plants initiates upon seed germination, radial growth is only primed during early ontogenesis in procambium cells and activated later by the vascular cambium ${ }^{1}$. Although it is not known how radial growth is organized and regulated in plants, this system resembles the developmental competence observed in some animal systems, in which pre-existing patterns of developmental potential are established early on ${ }^{2,3}$. Here we show that the initiation of radial growth occurs around early protophloem sieve element (PSE) cell files of the root procambial tissue in Arabidopsis. In this domain cytokinin signalling promotes expression of a pair of novel mobile transcription factors, PHLOEM EARLY DOF (PEAR1, PEAR2) and their four homologs (DOF6, TMO6, OBP2 and HCA2), collectively called PEAR proteins. The PEAR proteins form a short-range concentration gradient peaking at PSE and activating gene expression that promotes radial growth. The expression and function of PEAR proteins are antagonized by well-established polarity transcription factors, HD-ZIP III ${ }^{4}$, whose expression is concentrated in the more internal domain of radially non-dividing procambial cells by the function of auxin and mobile miR165/166. The PEAR proteins locally promote transcription of their inhibitory HD-ZIP III genes, thereby establishing a negative feedback loop that forms a robust boundary demarking the zone of cell divisions. Taken together, we have established a network, in which the PEAR - HD-ZIP III module integrates spatial information of the hormonal domains and miRNA gradients during root procambial development, to provide adjacent zones of dividing and more quiescent cells as a foundation for further radial growth.

Cambial growth in plants is initiated within the procambial tissues of the apical meristems through periclinal (i.e. longitudinal) divisions associated with formation of the vascular tissues xylem and phloem ${ }^{1}$ (Extended Data Fig. 1a). It has been established that during procambial development in Arabidopsis roots there are distinct domains for high auxin and cytokinin signalling, which mark the regions for further development of xylem and phloem/procambium, respectively ${ }^{5-8}$. To accurately map the spatial distribution of the periclinal divisions, we established a new nomenclature for the root procambial cells, including PSE-lateral neighbours (PSE-LN) as cells directly contacting both PSE and the pericycle, the outer procambial cells (OPC) as procambial cells adjacent to the pericycle but not contacting PSE, and SE-internal neighbours (PSE-IN) as cells located internal to and directly contacting PSE (Fig. 1a). Both the PSE cell and PSE-LN showed higher activity of periclinal cell division than the OPC and PSE-IN (Fig. 1b, Extended Data Fig. 1b-d and Supplementary Information). We observed virtually no periclinal divisions in metaxylem (MX) and internal procambial cells (IPC) (Fig. 1b). Furthermore, blocking symplastic transport genetically ${ }^{9}$ between the PSE and the surrounding cells results in a dramatic reduction in the number of cell files, not only in PSE lineage but also in the PSE-LN lineage (Extended Data Fig. 2a-e). Thus, the proliferative activity in procambium is centred on and around PSE and may involve symplastic intercellular signals.

By searching in silico for transcription factors enriched in early PSE $^{10}$ (Extended Data Fig. 3a), we identified a pair of DOF transcription factors ${ }^{11}$, PHLOEM-EARLY-DOF 1 (PEAR1)/DOF2.4 and PEAR2/DOF5.1 $1^{12}$ (Extended Data Fig. 3b). RNA in situ hybridization and transcriptional fusion constructs validated that both PEAR1 and PEAR2 are transcribed specifically in PSE cells (Fig. 1c and Extended Data Fig. 3d). However, fluorescent tagged versions of the PEAR proteins show localization not only in PSE but also in PSE neighbouring cells (PSE-LN and PSE-IN), indicating that these proteins move across short ranges via plasmodesmata (Fig. 1d, Extended Data Fig. 2f-g, 3d and 4a-d). 
We next investigated whether the loss-of-function of these genes would lead to a phenotype corresponding to the one observed when symplastic transport is compromised (Extended Data Fig 2c). However, we did not find such phenotype in single or double mutants corresponding to PEARI and PEAR2 (Extended Data Fig. 5a and b). We subsequently identified DOF1.1/OBP2 ${ }^{13}, D O F 3.2 / D O F 6^{14}$, DOF5.6/HCA2 $2^{15}$, and DOF5.3/TMO6 ${ }^{16}$ as additional PSE specific/abundantly expressed DOF genes with a broader gene product localization (Extended data Fig 3d). Furthermore, overexpression of any of these six loci results in an increased number of cell files (Extended Data Fig. 3c). In addition, we observed that DOF6, HCA2 and TMO6 are upregulated in pearl pear2 double mutant apparently as a compensation response (Extended Data Fig. 3e, see also Supplementary Information). Among the several higher order combinatorial mutants involving all six genes, we found the pearl pear2 tmo6 triple mutant to display reduced radial growth variably (Fig. 2a, c and f), while the corresponding three double mutants did not show this phenotype (Extended Data Fig. 5b). Furthermore, the pear 1 pear 2 dof6 tmo6 quadruple mutant results in all plants with further, uniformly reduced radial growth corresponding to the line with compromised symplastic trafficking (Fig. 2d, f and Extended Data Fig. 2c), indicating that these four mobile PEAR proteins play a major role in radial growth. In addition, the pearl pear 2 dof6 obp 2 hca 2 quintuple mutant resulted in a population of slowly elongating roots (around 30 per cent, $n=300$ ) with a reduction in radial growth (Fig. $2 b$ and $f$ ), whereas the corresponding five quadruple mutants for the five genes did not display a strong phenotype (Extended Data Fig. 5b). The introduction of obp 2 and hca 2 mutations into the pear 1 pear 2 dof6 tmo6 quadruple background (resulting in the pear1 pear2 dof6 tmo6 obp2 hca 2 hextuple mutant) did not result in further reduced radial growth (Fig. 2d-f), collectively suggesting a significant but minor contribution of $O B P 2$ and $H C A 2$. We were able to suppress the phenotype of the quintuple and/or hextuple mutants with all six genes (Extended Data Fig. 5c, d and see Supplementary Information). Collectively these data indicate that the mobile PEAR proteins redundantly control cell proliferation in and around PSE cells. Their effects are likely to be both cell autonomous and/or non-cell autonomous as several putatively direct target genes, including a central regulator of phloem formation SUPPRESSOR OF MAX2 1-LIKE3 $(S M X L 3)^{17}$, are expressed in both PSE and its surrounding cells (Fig. 2g-h and Extended Data Fig. 6, also see Supplementary Information). Moreover, ectopic expression of SMXL3 is sufficient to enhance periclinal cell divisions (Extended Data Fig. 6j).

Earlier studies have highlighted cytokinins in regulating procambial cell proliferation ${ }^{6,8}$. During root development, cytokinin signalling reporter, $p A R R 5: \because R F P e r^{18}$ is initially activated and maintained in PSE and its surrounding procambial cells, later becoming concentrated in the procambial cells neighbouring to the xylem cells, while auxin response is maintained in xylem domain ${ }^{7,8}$ (Extended Data Fig. 7a, and see Supplementary Information). Cytokinin signalling reporter partially overlaps with the PEARl transcriptional domain (Fig. 3a). Exogenous cytokinin application rapidly increased the level of some of the PEAR transcripts (Extended Data Fig. 7b), and sustained cytokinin treatment resulted in a radial expansion of PEAR expression domains (Extended Data Fig. 7c). Conversely, both PEARI and TMO6 transcription were highly reduced in the procambial tissue of cytokinin signalling loss-of-function mutant wooden-leg (wol) ${ }^{5,19}$ (Fig. 3b and Extended Data Fig. 7d) and in plants overexpressing $A R R 22^{20}$, an inhibitor of cytokinin signalling (Fig. $3 \mathrm{~d}$ and e). However, expression of both genes was restored by the induction of cytokinin signalling in wol (Fig. 3c and Extended Data Fig. 7d). In addition, we validated the requirement of cytokinin signalling for PEARI 
expression during embryogenesis (Extended Data Fig. 7e-r, and see Supplementary Information). Taken together, our results indicate that initiation of PEARl expression in early embryogenesis is independent of cytokinin signalling, but by the time the bisymmetric cytokinin pattern is formed at early heart stage, PEARl transcription is activated and maintained post-embryonically by cytokinins.

Almost no periclinal cell divisions were observed in the cells non-adjacent to the pericycle, including PSE-IN where both cytokinin response and PEAR protein are present (Fig. 1b-d and 3a), suggesting an inhibitory mechanism restricts PEAR function in the inner cells. We previously observed an increased cell number in the vascular tissue of quadruple loss-of-function mutant of the five Class III HOMEODOMAIN LEUCINE ZIPPER (HD-ZIP III) genes ${ }^{21,22}$ (Fig. 4a, d and g). These ectopic cell divisions occur in cells non-adjacent to the pericycle (Extended Data Fig. 8a-e). We observed high levels of three HD-ZIP III proteins, PHABULOSA (PHB), CORONA (CNA) and REVOLUTA (REV), in non-dividing procambial cells, IPC and PSE-IN, whereas their expression was absent in the actively dividing cells of the PSE and PSE-LN (Extended Data Fig. 8f-k). In this domain endodermal-derived mobile miR165/6 eliminates HD-ZIP III messenger RNA ${ }^{22,23}$, suggesting that HD-ZIP III inhibit periclinal cell divisions of PSE-IN by antagonizing the functions of co-localized PEAR proteins. This is further supported by our observation that overexpression of PEARl in the miRNA-resistant phb-1d mutant which has elevated levels of $\mathrm{PHB}^{22,23}$ is less effective than overexpressing PEARI in wild-type plants (Extended Data Fig. 81-o). Hence, to sharpen the boundary between dividing and non-dividing cells, the expression pattern of both HD-ZIP III and PEAR proteins must be tightly controlled.

Auxin is known to promote the xylem associated $H D$-ZIP III transcription ${ }^{24,25}$. However, $P H B$, $C N A$ and $R E V$ show broader expression of both transcriptional and translational reporters (Fig. 4h, Extended Data Fig. 8f-h and Extended Data Fig. 9), suggesting that other factors may enhance $H D$ ZIP III transcription in the peripheral region. Interestingly, we observed a significant reduction of CNA transcription in PSE-neighbouring cells in the pear quintuple background (Fig. 4h-j). In addition, PEARI overexpression enhanced the transcription of HD-ZIP III genes, especially in the central domain of vascular tissue (Extended Data Fig. 9). These data suggest that PEAR1 locally enhances HD-ZIP III transcription at PSE-neighbouring cells. As previous work has reported that PEAR1 has the potential to bind $H D-Z I P I I I$ promoters ${ }^{26,27}$, it is possible that these interactions are direct. As $H D$ ZIP III and PEARI show complementary expression patterns, we explored whether HD-ZIP III could regulate $P E A R 1$ transcription. $P E A R 1$ expression was severely attenuated in mutants showing elevated levels of HD-ZIP III such as $p h b-1 d$ and $s h r-2^{22}$ (Extended Data Fig.8p-t). Together these data suggest a feedback loop between HD-ZIP III and PEARI transcription.

Furthermore, to examine a possible effect of the HD-ZIP III on the mobile PEAR1 proteins, we measured the diffusion coefficient and movement pattern of PEAR1-GFP in wild type and in the $h d$-zip III quadruple mutant where PSE is formed in a triarch arrangement but PEARl transcription is restricted to PSE as observed in wild type (Extended Data Fig. 8u and w). We observed that the diffusion coefficient of PEAR1-GFP is significantly higher and the protein moves further in the mutant compared to wild type (Fig. 4k-m and Extended Data Fig. 4). To understand the significance of this enhanced PEAR1 movement, we analysed the cell proliferation pattern of combinatorial pear 1 pear 2 dof6 obp 2 hca 2 phb phv cna athb8 nonuple and pear 1 pear2 dof6 tmo6 obp 2 hca 2 phb phv cna athb8 decuple loss-of-function mutants. We found that these mutants showed a reduced number of periclinal cell divisions in the vascular cells both adjacent to and non-adjacent to the pericycle compared to the 
hd-zip III quadruple mutant (Fig. 4a-g and Extended Data Fig. 8d-e). This indicates that HD-ZIP III inhibit periclinal cell division partially through inhibiting PEAR1 movement to position the cell division zone around phloem.

In order to further conceptualize the observed interactions between PEAR and HD ZIP III and test the capacity of this network to generate sharp boundaries, we incorporated the PEAR factors into a spatially one dimensional network model with HD-ZIP III, miR165/6, auxin and cytokinin as defined in previous theoretical studies ${ }^{8,28,29}$ (Supplementary Modelling Information). The model is defined on a line in one spatial dimension representing 3, 4 or 5 cells from the centre of the xylem axis to the outer edge of the PSE cell (Extended Data Fig. 10a-d and Supplementary Modelling Information). One particularly interesting aspect of the system is that the network involves dual negative feedback loops, in which HD-ZIP III transcription is activated by PEAR1 (Interaction (1) in Extended Data Fig.10c), while in turn both PEARI transcription and protein movement are inhibited by HD-ZIP III (Interaction (2) and (3), respectively in Extended Data Fig.10c). We ran simulations exploring the steady state patterns created in networks with the above interactions and in scenarios when one of the interactions was missing (Extended Data Fig. 10d-h). Based solely on two inputs imposed at the margins, auxin (xylem) and miR165/6 (outer margin), the model predicts the spatial distribution of cytokinin, as well as PEAR and HD-ZIP III proteins (Extended Data Fig. 10d-h). The version of the model incorporating all three interactions (i.e repressing both the transcription and movement of PEAR) results in the sharpest gradients of PEAR and HD-ZIP III proteins (Extended Data Fig. 10f) with both PEAR1 protein and HD-ZIP III localized within the PSE-IN, consistent with experimental observations (Extended Data Fig. 10e). To our knowledge this is the first report of a role for the dual regulation of both transcription and movement of a developmental regulator in sharpening boundaries.

Collectively our research has uncovered a regulatory network involving the dual regulation of gene transcription and protein movement, in which the spatial distribution of phytohormones and small RNA is decoded into the activity of two functionally antagonistic sets of transcription factors, PEAR and HD-ZIP III, during root procambial development (Extended Data Fig. 10i). The mobile PEAR factors promote cell proliferation around the two early protophloem sieve element cell files, which constitute two new organizers just proximal to the quiescent centre. These organizers surround a more quiescent central zone defined by the HD-ZIP III factors. In this way, the PEAR - HD-ZIP III module specifies a lateral meristem within an apical meristem and as such, forms a foundation for further cambial development ${ }^{30}$. Therefore, in the future it will be interesting to determine how extensively this procambial pathway also contributes to ontogenetically late processes such as wood and storage organ formation in the crop species.

\section{Acknowledgements}

We thank E. Scarpella, ABRC and NASC for materials, Katja Kainulainen, Karolina Blajecka, Mikko Herpola and Alessandro Mainardi for technical assistance, Natalie Clark for assisting with the scanning FCS analysis, Jenny Jansen for technical assistance with microarray hybridizations, Oleg Kambur and Lothar Kalmbach for assistance in generating the heatmap, and D. Weijers and T. Kakimoto for helpful discussions, A. Groenheide, E. Cornelissen, M. Chu, A. Korppoo and Alba Rodriguez Diez for technical support. This works was supported by Finnish Centre of Excellence in Molecular Biology of 
Primary Producers (Academy of Finland CoE program 2014-2019) decision \#271832, the Gatsby Foundation [GAT3395/PR3)], University of Helsinki [award 799992091] and the European Research Council Advanced Investigator Grant SYMDEV [No. 323052] to Y. H., a NSF-BBSRC MCSB 1517058 to R.S. and Y.H., an ERC Consolidator grant (PLANTSTEMS), a Heisenberg Professorship of the German Research Foundation (DFG, GR2104/5-1) and the SFB 873 (DFG) to T.G., the Netherlands Organization for Scientific Research (NWO; VIDI-864.13.001) and The Research Foundation - Flanders (FWO; Odysseus II G0D0515N and 12D1815N) to W.S. and B.D.R., respectively, a JSPS postdoctoral fellowship for research abroad and JSPS KAKENHI Grant [17K15138] to S.M., Swiss National Science Foundation Postdoc Mobility Grant (P300P3_147894) to P.R., a JSPS Research Fellowship for Young Scientists and JSPS KAKENHI Grant [JP16J00131] to K.T., Bayer Science and Education Foundation, German Academic Exchange Service (DAAD) to B.B., The Finnish Academy of Science to J. O. H., Herchel Smith Postdoctoral Research Fellowship (Herchel Smith Fund) to S.O.

\section{Author contributions}

S.M., P.R. and I.S. contributed equally to this work. S.M. characterized the molecular interactions among PEAR and HD-ZIP III module. P.R. identified and quantified phenotype in the PEAR loss of function mutants with help of B.B. I.S. determined phloem specific DOFs and their downstream genes with input from B.D.R., W.S., M.B. and G. H. K.T. characterized PEAR-HD-ZIP III combinatorial mutants. B.B. generated tmo6 CRISPR mutants. J.H. performed in situ hybridization. N.M. and A.B. designed and performed computational modelling. H.H. produced CRE1 inducible line. S.O. assisted in the microarray experiments. K.H. and K.N. produced HD-ZIP III reporter lines. O.S. and A.P.M. provided the destination vector $\mathrm{pSm} 43 \mathrm{GW}$. R.S. and A.P.M. provided the $p A R R 5: \because R F P e r$ line. E.S.W., Y.K., T.G. and C.M. shared informative non-published data. R.S. analysed diffusion coefficient of PEAR1-GFP with P.R. B.D.R. and Y.H. participated in experimental design. S.M. and Y.H. wrote the manuscript and all authors commented on the manuscript. B.D.R. and Y.H. are co-corresponding authors.

Competing interests The authors declare no competing interests.

\section{Additional information}

Extended data is available for this paper at https://doi.org/10.1038/s41586- 018-0839-y.

Supplementary information is available for this paper at https://doi.org/ 10.1038/s41586-018-0839y.

Reprints and permissions information is available at http://www.nature.com/ reprints.

Correspondence and requests for materials should be addressed to B.R. or Y.H.

\section{References}

1 Esau, K. Vascular differentiation in plants. (Holt, Rinehart and Winston, 1965).

2 Crick, F. H. \& Lawrence, P. A. Compartments and polyclones in insect development. Science 189, 340-347 (1975).

3 Dickson, B., Sprenger, F. \& Hafen, E. Prepattern in the developing Drosophila eye revealed by an 
activated torso--sevenless chimeric receptor. Genes Dev 6, 2327-2339 (1992).

McConnell, J. R. et al. Role of PHABULOSA and PHAVOLUTA in determining radial patterning in shoots. Nature 411, 709-713, doi:10.1038/35079635 (2001).

Mähönen, A. P. et al. A novel two-component hybrid molecule regulates vascular morphogenesis of the Arabidopsis root. Genes Dev 14, 2938-2943 (2000).

Mähönen, A. P. et al. Cytokinin signaling and its inhibitor AHP6 regulate cell fate during vascular development. Science 311, 94-98, doi:10.1126/science.1118875 (2006).

Bishopp, A. et al. A mutually inhibitory interaction between auxin and cytokinin specifies vascular pattern in roots. Curr Biol 21, 917-926, doi:10.1016/j.cub.2011.04.017 (2011).

De Rybel, B. et al. Plant development. Integration of growth and patterning during vascular tissue formation in Arabidopsis. Science 345, 1255215, doi:10.1126/science.1255215 (2014).

Vatén, A. et al. Callose biosynthesis regulates symplastic trafficking during root development. Dev Cell 21, 1144-1155, doi:10.1016/j.devcel.2011.10.006 (2011).

Brady, S. M. et al. A high-resolution root spatiotemporal map reveals dominant expression patterns. Science 318, 801-806, doi:10.1126/science.1146265 (2007).

Yanagisawa, S. The Dof family of plant transcription factors. Trends Plant Sci 7, 555-560 (2002).

2 Kim, H. S. et al. The DOF transcription factor Dof5.1 influences leaf axial patterning by promoting Revoluta transcription in Arabidopsis. Plant J 64, 524-535, doi:10.1111/j.1365-313X.2010.04346.x (2010).

3 Skirycz, A. et al. DOF transcription factor AtDof1.1 (OBP2) is part of a regulatory network controlling glucosinolate biosynthesis in Arabidopsis. Plant $J$ 47, 10-24, doi:10.1111/j.1365313X.2006.02767.x (2006).

4 Rueda-Romero, P., Barrero-Sicilia, C., Gómez-Cadenas, A., Carbonero, P. \& Oñate-Sánchez, L. Arabidopsis thaliana DOF6 negatively affects germination in non-after-ripened seeds and interacts with TCP14. $J$ Exp Bot 63, 1937-1949, doi:10.1093/jxb/err388 (2012).

Guo, Y., Qin, G., Gu, H. \& Qu, L. J. Dof5.6/HCA2, a Dof transcription factor gene, regulates interfascicular cambium formation and vascular tissue development in Arabidopsis. Plant Cell 21, 3518-3534, doi:10.1105/tpc.108.064139 (2009).

Schlereth, A. et al. MONOPTEROS controls embryonic root initiation by regulating a mobile transcription factor. Nature 464, 913-916, doi:10.1038/nature08836 (2010).

Wallner, E. S. et al. Strigolactone- and Karrikin-Independent SMXL Proteins Are Central Regulators of Phloem Formation. Curr Biol 27, 1241-1247, doi:10.1016/j.cub.2017.03.014 (2017).

Siligato, R. et al. MultiSite Gateway-Compatible Cell Type-Specific Gene-Inducible System for Plants. Plant Physiol 170, 627-641, doi:10.1104/pp.15.01246 (2016).

9 Mähönen, A. P. et al. Cytokinins regulate a bidirectional phosphorelay network in Arabidopsis. Curr Biol 16, 1116-1122, doi:10.1016/j.cub.2006.04.030 (2006).

Kiba, T., Aoki, K., Sakakibara, H. \& Mizuno, T. Arabidopsis response regulator, ARR22, ectopic expression of which results in phenotypes similar to the wol cytokinin-receptor mutant. Plant Cell Physiol 45, 1063-1077, doi:10.1093/pcp/pch128 (2004).

Prigge, M. J. et al. Class III homeodomain-leucine zipper gene family members have overlapping, antagonistic, and distinct roles in Arabidopsis development. Plant Cell 17, 61-76, doi:10.1105/tpc.104.026161 (2005). 

Nature 465, 316-321, doi:10.1038/nature08977 (2010).

Miyashima, S., Koi, S., Hashimoto, T. \& Nakajima, K. Non-cell-autonomous microRNA165 acts in a dose-dependent manner to regulate multiple differentiation status in the Arabidopsis root. Development 138, 2303-2313, doi:10.1242/dev.060491 (2011).

24 Baima, S. et al. The expression of the Athb-8 homeobox gene is restricted to provascular cells in Arabidopsis thaliana. Development 121, 4171-4182 (1995).

Donner, T. J., Sherr, I. \& Scarpella, E. Regulation of preprocambial cell state acquisition by auxin signaling in Arabidopsis leaves. Development 136, 3235-3246, doi:10.1242/dev.037028 (2009).

O'Malley, R. C. et al. Cistrome and Epicistrome Features Shape the Regulatory DNA Landscape. Cell 166, 1598, doi:10.1016/j.cell.2016.08.063 (2016).

Gaudinier, A. et al. Enhanced Y1H assays for Arabidopsis. Nat Methods 8, 1053-1055, doi:10.1038/nmeth.1750 (2011).

Muraro, D. et al. Integration of hormonal signaling networks and mobile microRNAs is required for vascular patterning in Arabidopsis roots. Proc Natl Acad Sci $U S A$ 111, 857-862, doi:10.1073/pnas.1221766111 (2014).

29 Mellor, N. et al. Theoretical approaches to understanding root vascular patterning: a consensus between recent models. J Exp Bot 68, 5-16, doi:10.1093/jxb/erw410 (2017).

De Rybel, B., Mähönen, A. P., Helariutta, Y. \& Weijers, D. Plant vascular development: from early specification to differentiation. Nat Rev Mol Cell Biol 17, 30-40, doi:10.1038/nrm.2015.6 (2016).

\section{Methods}

No statistical methods were used to predetermine sample size. The experiments were not randomized, and investigators were not blinded to allocation during experiments and outcome assessment. Experiments were repeated at least twice. All experiments were repeated successfully.

\section{Plant materials and growth condition}

Arabidopsis thaliana lines used in this study were either in Columbia or Landsberg erecta background. The following alleles were obtained from the publicly available collections: pear1 (CSHL_GT8483) in Ler, pear2 (SALK_088165) in Col-0, obp2 (SK24984) in Col-4, dof6 (Wiscseq_Ds_Llox351c08) in Col-0, hca2 (GK-466B10) in Col-0. Knock-out alleles of TMO6 were generated using CRISPRCas9 technology as previously described ${ }^{31}$. The following protospacer target sequence was selected as it had no predicted off-site targets and allowed screening via NheI restriction using the CRISPR-P web tool $^{32}$. The Protospacer adjacent motif is underlined: GGACACCTGAGAGCTAGCTCCGG. Successful mutagenesis was confirmed via Sanger sequencing in plants of the T2 and T3 generation that no longer carried the Cas9 transgene. Four TMO6 mutant alleles were identified: tmo6-1 (+A), tmo6-2 (+T), tmo6-3 (deletion of $5 \mathrm{bp}$ and at the same time insertion of $26 \mathrm{bp}$ ) and tmo6-4 (-5 bp) (Extended Data Fig. 5a). The alleles tmo6-1, -2, and -3 were found in the pear hextuple mutant and caused the pear hextuple phenotype, while tmo6-4 was found in the tmo6 single mutant, respectively. The genotyping primers for these mutants are listed in Supplementary Table 1. hd-zip III quadruple 
(phb phv cna athb8) was described previously ${ }^{21}$. Plant growth conditions were described previously ${ }^{5}$.

\section{Histological analysis}

Primary roots of vertically grown 4 to 5-day-old seedlings were used for histological analyses. For confocal imaging, root samples were stained with propidium iodide (PI), aniline blue (AB) or SCRI Renaissance 2200 (SR2200) (Renaissance Chemicals, UK). The method of PI and AB staining were described previously ${ }^{9,33}$. For SR2200 stain, root samples were fixed in SR2200 solution (4\% paraformaldehyde, $0.1 \%$ (v/v) SR2200 in PBS buffer (pH7.4)). Then samples were washed with PBS buffer and transferred into the ClearSee solution ${ }^{34}$. Confocal imaging was performed on Leica TCS SP5, Leica TCS SP8, Leica TCS SP5 II HCS-A or Nikon C2 CLSM using a solid state blue laser (480nm) for GFP, a green laser (514nm) for VENUS, a lime laser (DPSS 561nm) for RFP and PI, and a UV laser (diode 405nm) for SR2200. Transverse plastic sections of root were performed as described previously ${ }^{5}$. For histological analyses of embryo, dissected embryos were mounted in SR2200 solution and visualized by the confocal microscopy.

\section{Mapping of the position of periclinal cell divisions}

A series of 2D confocal images of Arabidopsis root vascular tissue were recorded at $0.5 \mu \mathrm{m}$ intervals using Nikon C2 CLSM or Leica TCS SP8. Cross section images in each developmental stage were created by ImageJ software from a series of 2D confocal images, and the cell segmentation was done using CellSeT ${ }^{35}$. For more information, see Supplementary Information.

\section{Box plots}

Box plots were created with standard box blot setting (the first and third quartiles, split by the median; whiskers extend to a maximum of $1.5 \times$ interquartile range (IQR) beyond the box.) Outliers are indicated as black dots.

\section{DNA constructs and transgenic plants}

Most of transgenic constructs were produced by using Gateway or multisite Gateway system (Invitrogen) as described previously ${ }^{18}$. To generate the transcriptional fusion constructs with GFP-GUS each promoter sequence was cloned into pDONR221 and fused to GFP-GUS coding sequence in the destination vector $\mathrm{pBGWFS7}$ by normal LR reaction. For other transcriptional fusion constructs, including pPEAR1::VENUSer, pPEAR2::VENUSer, pAHA3::RFPer, pOBP $2:: V E N U S e r$, pHCA2::RFPer, pTMO6::RFPer and $p R E V:: R F P e r$ and the transcriptional fusion constructs of PEAR1/PEAR2 downstream genes, each promoter was cloned into pDONRP4_P1R, and assembled with the coding sequence of fluorescent reporter (VENUSer or RFPer) and terminator into the destination vectors, $\mathrm{pHm} 43 \mathrm{GW}$ (Hygromycin resistant), $\mathrm{pBm} 43 \mathrm{GW}$ (Basta resistant) or by multisite Gateway system. To produce the transcriptional fusion constructs of HD-ZIP III, including PHB and CNA, each promoter was inserted upstream of the GAL4:VP16 (GV) coding region of pBIB-UASGFPer-NtADH5'-GV vector ${ }^{36}$. For most of the translational fusion constructs of PEAR genes, except for $p P E A R 1:: P E A R 1-G F P$, each promoter was cloned into the first-box vector pDONRP4_P1R, and each coding sequence was cloned into vector pDONR221, thereafter each promoter and coding sequence were assembled with pDONR P2R_P3-terminator/reporter into pHm43GW, pBm43GW or 
pFR7mGW by multisite Gateway system ${ }^{18}$. To generate other translational fusion constructs, including pPEAR1::PEAR1-GFP, $p C N A:: C N A-G F P, \quad p A T H B 8:: A T H B-G F P$ and $p R E V:: R E V-G F P$, each genomic fragment which contains promoter, coding and its 3 ' region, was cloned into pAN19 vector. Then GFP coding sequence was fused to C-terminus of each coding sequence. Finally, each translational fusion sequence was inserted into the modified pBIN19 vector with Basta resistance ${ }^{23}$. For the overexpression construct, including PEAR genes and $C R E 1$, the coding sequence of each genes was assembled with stele-specific estradiol-inducible promoter (pCRE1[XVE]) into $\mathrm{pHm} 43 \mathrm{GW}$ or pBm43GW by the Multisite Gateway system described previously ${ }^{18}$. To construct pPEAR1[XVE]::icals3m, 1.5kb PEARl promoter was cloned into p1R4-ML:XVE vector ${ }^{18}$, and assembled with icals $3 \mathrm{~m}$ sequence into $\mathrm{pBm} 43 \mathrm{GW}^{9}$. The primers for DNA construction and the list of plasmids are shown in Supplementary Table 1.

\section{In situ hybridization}

Amplified fragments of $P E A R 1, P E A R 2$ and $O B P 2$ were cloned into pGEM-T Easy (Promega) vector and fragments of DOF6, HCA2, TMO6 into pCR-Bunt II-TOPO vector (Invitrogen) following manufacturer's instructions. In order to obtain antisense probes, plasmids were first linearized by restriction enzyme treatment: MluI for PEAR1 and OBP2, ScaI for PEAR2, HindIII for TMO6 and $D O F 1$, and $X b a \mathrm{I}$ for $H C A 2$ were used. Linearized plasmids were digoxigenin (DIG) labelled using DIG RNA Labelling Kit (Roche) following manufacturer's instructions. For PEAR1, OBP2, TMO6 and DOF1, T7 RNA polymerase and for PEAR2 and HCA2, SP6 RNA polymerase were used. mRNA detection on a whole-mount seedlings was performed as described ${ }^{37}$. Images were taken with Zeiss Axioimager microscope with either 20x or 40x objective.

\section{Transcriptome analysis}

Targets of PEAR1 and PEAR2 were identified by analysing transcriptional changes after dexamethasone (DEX) treatment of pRPS5A::PEAR1-GR and pRPS5A::PEAR2-GR. To identify putative direct targets, DEX treatment was also performed with cycloheximide (CHX), which inhibits protein synthesis and therefore activation of indirect targets. 3-day-old seedlings were grown on control medium and transferred to medium containing $10 \mu \mathrm{M}$ DEX or $10 \mu \mathrm{M}$ DEX and $10 \mu \mathrm{M}$ CHX for $2 \mathrm{~h}$, after which root tips were collected and RNA extraction was performed. Total RNA (100 ng) was labelled using GeneChip WT PLUS Reagent Kit (Thermo Fisher Scientific) and hybridized to GeneChip Arabidopsis Gene 1.1 ST array plates (Affymetrix). Sample labelling, hybridization to chips, and image scanning were performed according to the manufacturer's instructions. Microarray analysis was performed as previously described to yield significantly up-regulated genes $(>1.0 \text {-fold; } \mathrm{P}<0.05)^{8}$. Venn diagram of significantly up-regulated genes was made using Venny 2.1 on-line program (http://bioinfogp.cnb.csic.es/tools/venny_old/venny.php). Previously published root spatiotemporal expression data was used to make a heatmap to visualize predicted expression patterns of all PEAR1 and PEAR2 targets ${ }^{10}$. To have relative expression values for every gene in different root cell types and developmental stages, values for every gene were normalized based on its highest expression in one of the cell types. Heatmap was generated using R with gplots R-package ${ }^{38}$. The transcriptomics data files are submitted to GEO (accession number GSE115183). 


\section{Reporter analysis of PEAR1/2 downstream genes}

When selecting genes for reporter analysis, putative direct targets were preferred. Significantly more direct targets were identified for $P E A R 2$, and therefore those are overrepresented. Other considerations were how strongly they were upregulated, as well as their predicted expression pattern. Expression in early procambium or early phloem and procambium was preferred. AT1G49230, AT1G15080, AT3G16330, AT4G00950 and SMXL3 are putative direct targets of PEAR2 and with predicted expression in early phloem/procambium. AT3G54780 was chosen because it is a putative direct target of both PEAR1 and PEAR2, although no predicted expression data was available. AT1G09460, a direct target of PEAR2 and a target of PEAR1, was chosen because it was induced very strongly by both genes, although predicted to be expressed only very weakly in phloem/procambium.

\section{Quantitative RT-PCR analysis}

qRT-PCR analyses were performed as described previously ${ }^{39}$. Cytokinin treatment was done with $10 \mu \mathrm{M}$ 6-Benzylaminopurine (BA), and experiments were performed in three biological repeats and each of these with 3 technical repeats. RNA was extracted with the RNeasy kit (QIAGEN). Poly(dT) cDNA was prepared from $1 \mu \mathrm{g}$ of total RNA with an iScript cDNA Synthesis Kit (Biorad) and analysed on a CFX384 Real-Time PCR detection system (BioRad) with iQ SYBR Green Supermix (BioRad) according to the manufacturer's instructions. Expression levels were normalized to those of EEF1 $\alpha$ and CDKA1;1. The primers are listed in Supplementary Table 1.

\section{Phloem transport assay}

The phloem translocation was judged by the transport and unloading of 5(6) Carboxyfluorascein diacetate (CFDA) as describe ${ }^{40}$. After application of the dye, plants were kept in agar plates and only placed on regular cover slips for imaging.

\section{Raster image correlation spectroscopy (RICS)}

To determine the rate of movement of GFP-labeled PEAR1 protein in wild type and hd-zip III quadruple (phb phv cna athb8) mutant background, Raster image correlation spectroscopy (RICS) was performed according to previous work ${ }^{41-43}$. Images were collected using a Zeiss 880 confocal microscope. Frames of $256 \times 256$ pixels were acquired using a raster scan with a dwell time of 8.19 $\mu$ sec pixel $^{-1}$ at a pixel size of either $100 \mathrm{~nm}$ for 100 frames resulting in a line scan of $5.035 \mathrm{~ms}$. Diffusion coefficients were derived using the SimFCS software (https://www.lfd.uci.edu/globals/) ${ }^{44}$ from GFPlabeled PEAR1 vascular cells within the first $70 \mu \mathrm{m}$ from the QC. Specifically, 18 observations from WT and 30 for the hd-zip III quadruple (phb phv cna athb8) mutant background were used for the RICS analysis using the SimFCS software The RICS algorithm by comparing the intensity fluctuations of one pixel to the fluctuations of the pixels next to it and the fluctuations of one pixel to itself over time, produces a spatio-temporal Auto Correlation Function (ACF) that captures the fluorescence dynamics of the particles in the volume ${ }^{44,45}$. The ACF is decomposed into two correlation functions that depend on $\xi$ (the spatial lag in $x$ ) and $\psi$ (the spatial lag in $y$ ). The first correlation function, $S(\xi, \psi)$, calculates the spatio-temporal correlation due to the scanning of the microscope. The second correlation function, $G(\xi, \psi)$, calculates the spatio-temporal correlation due to particles diffusing in the medium. The ACF, $G_{S}(\xi, \psi)$, takes both of these correlations into account by multiplying them: 
$G_{S}(\xi, \psi)=S(\xi, \psi)^{*} G(\xi, \psi)$. The functions are constructed assuming that the distribution of fluorescence intensities follows a 3D Gaussian distribution. The decomposition of the ACF into two parts allows RICS to distinguish random, Brownian motion from diffusing particles in the medium ${ }^{45}$. The software fits the RICS-ACF using the pixel dwell time, pixel size, line scan and the Point Spread Function (PSF) bean waist of $0.241 \mathrm{~nm}$ as previously obtained ${ }^{41}$ and returns the diffusion coefficient of the protein. The diffusion coefficient returned results in the ACF curve that best fits the data. Goodness of fit is determined by comparing the residuals to the amplitude of the $\mathrm{ACF}^{41-45}$.

\section{Mathematical model}

The mathematical model is formulated as a set of ordinary differential equations describing the set of interactions shown in Figure 4o, defined on a one-dimensional array of discrete spatial compartments representing a cross-section of root tissue. The spatial subdivisions may represent either cell or cell wall compartments, with multiple compartments per cell so that intracellular resolution is present within the model. Three, four or five cells are simulated, from the centre of the stele at the xylem axis to the edge of the stele where phloem is formed. The model is implemented as a single stand-alone text file using Python 2.7 plus the open source libraries Scipy, from which the 'odeint' function was used to solve the differential equations, and Matplotlib, which was used to plot the figures. See Supplementary Modelling Information for more details.

\section{Code availability}

The code for mathematical model is available on request.

\section{Data availability}

All lines and data supporting the findings of this study are available from the corresponding author upon request. The microarray data files are available at Gene Expression Omnibus (GEO) (accession no. GSE115183).

\section{Methods references}

31 Fauser, F., Schiml, S. \& Puchta, H. Both CRISPR/Cas-based nucleases and nickases can be used efficiently for genome engineering in Arabidopsis thaliana. Plant $J$ 79, 348-359, doi:10.1111/tpj.12554 (2014).

32 Lei, Y. et al. CRISPR-P: a web tool for synthetic single-guide RNA design of CRISPR-system in plants. Mol Plant 7, 1494-1496, doi:10.1093/mp/ssu044 (2014).

33 Wysocka-Diller, J. W., Helariutta, Y., Fukaki, H., Malamy, J. E. \& Benfey, P. N. Molecular analysis of SCARECROW function reveals a radial patterning mechanism common to root and shoot. Development 127, 595-603 (2000). 

to extract and analyze structured networks of plant cells from confocal images. Plant Cell 24, 13531361, doi:10.1105/tpc.112.096289 (2012).

Hashimoto, K., Miyashima, S., Sato-Nara, K., Yamada, T. \& Nakajima, K. Functionally Diversified Members of the MIR165/6 Gene Family Regulate Ovule Morphogenesis in Arabidopsis thaliana. Plant Cell Physiol 59, 1017-1026, doi:10.1093/pcp/pcy042 (2018).

Hejátko, J. et al. In situ hybridization technique for mRNA detection in whole mount Arabidopsis samples. Nat Protoc 1, 1939-1946, doi:10.1038/nprot.2006.333 (2006).

38 Wickham, H. \& Sievert, C. ggplot2 : elegant graphics for data analysis. Second edition. edn, (Springer, 2016).

39 De Rybel, B. et al. A bHLH complex controls embryonic vascular tissue establishment and indeterminate growth in Arabidopsis. Dev Cell 24, 426-437, doi:10.1016/j.devcel.2012.12.013 (2013). 40 Oparka, K. J., Duckett, C. M., Prior, D. A. M. \& Fisher, D. B. Real - time imaging of phloem unloading in the root tip of Arabidopsis. The Plant Journal 6, 759-766, doi:doi:10.1046/j.1365313X.1994.6050759.x (1994).

41 Clark, N. M. et al. Tracking transcription factor mobility and interaction in Arabidopsis roots with fluorescence correlation spectroscopy. Elife 5, doi:10.7554/eLife.14770 (2016).

Clark, N. M. \& Sozzani, R. Measuring Protein Movement, Oligomerization State, and ProteinProtein Interaction in Arabidopsis Roots Using Scanning Fluorescence Correlation Spectroscopy (Scanning FCS). Methods Mol Biol 1610, 251-266, doi:10.1007/978-1-4939-7003-2_16 (2017).

O'Lexy, R. et al. Exposure to heavy metal stress triggers changes in plasmodesmatal permeability via deposition and breakdown of callose. $J$ Exp Bot 69, 3715-3728, doi:10.1093/jxb/ery171 (2018). Digman, M. A. et al. Measuring fast dynamics in solutions and cells with a laser scanning microscope. Biophys J 89, 1317-1327, doi:10.1529/biophysj.105.062836 (2005).

Digman, M. A. \& Gratton, E. Analysis of diffusion and binding in cells using the RICS approach. Microsc Res Tech 72, 323-332, doi:10.1002/jemt.20655 (2009). 



Figure 1| Periclinal cell divisions are centred around PSE, a domain highlighted by mobile PEAR transcription factors.

a, Schematic representation of procambial cells based on the position relative to PSE (red) and outer pericycle (gray). PSE neighbouring cells are classified as PSE lateral neighbour (PSE-LN, orange), a cell adjacent to both PSE and pericycle, or PSE internal neighbour (PSE-IN, darkgreen), a cell adjacent to PSE but not pericycle. Intervening procambial cells are classified as outer PC (OPC, yellow), a procambial cell adjacent to pericycle, or internal PC (IPC), a procambial cell non-adjacent to pericycle. PX and MX represent protoxylem and metaxylem, respectively. b, Number of periclinal cell divisions in each cell during procambial development ( 273 division events in total from 13 independent roots, also see Supplementary Information). PSE and PSELN exhibited higher proliferative activity. Bar graphs represent mean. Error bars are s.d. Dots, individual data points. c, Expression of pPEAR1::GFP-GUS ( $n=17)$ exhibits a highly PSE-specific expression pattern in the vascular tissue, though a residual level of GFP signal is observed in PSE-IN, most likely due to the retention of fluorescent protein after the division of PSE. d, Expression of the translational fusion of PEAR1 to GFP $(n=15)$. Fluorescent signal is observed not only in PSE but also in its neighbouring cells, including PSE-LN and PSE-IN. In c and $\mathbf{d}, n$ represents independent biological samples. White, darkgreen, orange arrowheads and asterisks indicate PSE, PSE-IN, PSE-LN and protoxylem, respectively. Scale bars, $25 \mu \mathrm{m}$. 

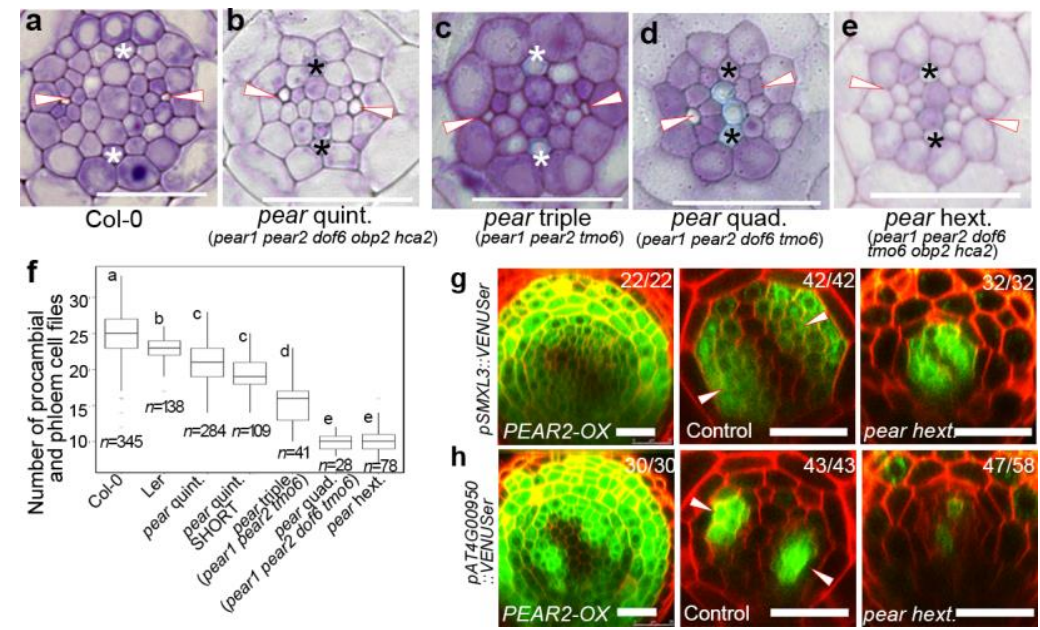

Figure 2| PEAR genes activate periclinal cell division by controlling downstream genes in non-cell autonomous manner.

a-e, Cross-section of wild type (a), pear quintuple (pear1 pear2 dof6 obp2 hca2) (b), pear triple (pear1 pear2 tmo6) (c), pear quadruple (pear1 pear2 dof6 tmo6) (d) and pear hextuple (pear1 pear2 dof6 obp2 hca2 tmo6) (e), respectively. Each image is representative of independent biological samples analysed in f. f, Number of procambial and phloem cell files in wild type and pear combinatorial mutants. Values were calculated from root cross sections at the differentiation zone. Boxplot centres show median. For more information on boxplots, see Methods. Statistically significant differences between groups were tested using Tukey's HSD test $p<0.05$. For individual $P$ values, see Supplementary Table 3. $n$, independent biological samples. g-h, Expression of selected PEAR1/2 downstream genes in wild type, PEAR2 overexpression plant and pear hextuple mutant. SMXL3 is expressed in phloem and procambial tissue, whose expression is induced by PEAR2 overexpression, but not altered in pear hextuple. AT4G00950 gene is expressed in PSE and its neighbouring cells, whose expression is induced by PEAR2 overexpression and reduced in pear hextuple mutant. Number in each panel indicates samples with similar results of the total independent biological samples analysed. White arrowheads, PSE. Asterisks, protoxylem. Scale bars, $25 \mu \mathrm{m}$. 

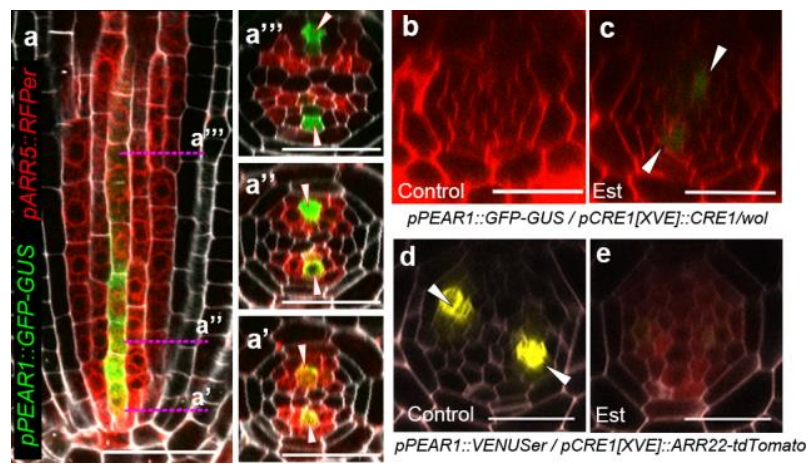

Figure 3 | Cytokinin signalling triggers PEAR1 expression.

a, Expression of $A R R 5$ and PEAR1 overlaps at the initial stage (a') and early proliferative phase (a"), $n=15$. b-c, Transcription of PEAR1 in wol root, which is conditionally rescued by CRE1 induction (Est, estradiol treated). PEAR1 transcription is severely reduced in the condition with attenuated cytokinin response ( $\mathbf{b}, n=6)$, and is restored after three days induction of CRE1 (c), arrowheads, $n=6)$. d-e, PEAR1 transcription is down-regulated after 48 hours of $A R R 22$ induction. $n=5$ and 7 , respectively. In a-e, $n$ represents independent biological samples. White arrowheads, PSE. Scale bars, $25 \mu \mathrm{m}$. 

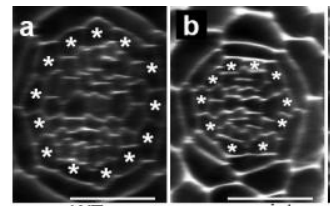

e

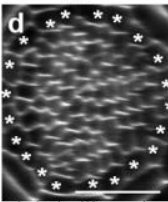

hd-zip III qua
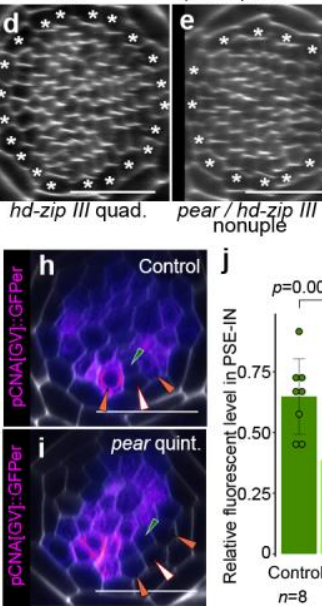



pear hext.

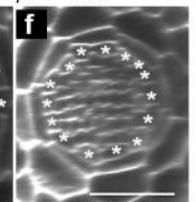$$
\text { . }
$$

$0-$


Figure 4| Antagonistic function of PEAR1 and HD-ZIP III sharpens the boundary between dividing and non-dividing cells.

a-f, An optical cross-section image of vascular tissue in wild type (a), pear quintuple (b), pear hextuple (c), hd-zip III quadruple (d), pear hd-zip III nonuple (e) and pear hd-zip III decuple mutant (f). Asterisks indicate pericycle cells. Each image is representative of independent biological samples analysed in $\mathbf{g}$. $\mathbf{g}$ Quantification of vascular cell number. In the analysis of pear quintuple and pear hd-zip III nonuple, a population of slowly elongating roots is selected as described in Fig. 2f. $\mathbf{h}-\mathbf{i}$, Expression of CNA transcriptional reporter in the control ( $h$, the heterozygous pear quintuple, $n=4$ ) and pear quintuple background $(\mathbf{i}, n=3)$. $\mathbf{j}$, Fluorescent level in PSE-IN is significantly reduced in pear quintuple. $n$ represents individual measurements across 4 or 3 independent biological samples, respectively. $\mathbf{k}-I$, PEAR1-GFP localization in wild type ( $\mathbf{k}, n=19)$ and $h d$-zip III quadruple mutant (I, $n=17)$. PEAR1-GFP is broadly localized even in IPC in $h d$-zip III quadruple (I, light-green arrowheads). $\mathbf{m}$, Average diffusion coefficient of PEAR1-GFP in wild-type and hd-zip III quadruple roots obtained by performing Raster Image Correlation Spectroscopy (RICS). In g-i and $\mathbf{k}-\mathbf{m}, \boldsymbol{n}$ represents independent biological samples. In $\mathbf{g}$, boxplot centres show median. Statistically significant differences were tested using Tukey's HSD test $p<0.05$. For individual $P$ values, see Supplementary Table 3. In $\mathbf{j}$ and $\mathbf{m}$, bar graphs represent mean. Error bars are s.d. (j) or s.e.m. (m). $P$ values were calculated by two-sided Student's t-test (j) or Mann-Whitney $U$ test (m). Dots, individual data points. White, dark-green, orange, light-green arrowheads indicate PSE, PSE-IN, PSE-LN and IPC, respectively. Scale bars, $25 \mu \mathrm{m}$. 




\begin{tabular}{|c|c|}
\hline Protoxylem (PX) $\stackrel{\underset{x}{x}}{=}$ & Protophloem sieve element (PSE) \\
\hline Metaxylem (MX) & Metaphloem sieve element (MSE) \\
\hline Intervening proc & Comp \\
\hline
\end{tabular}

Vascular tissue



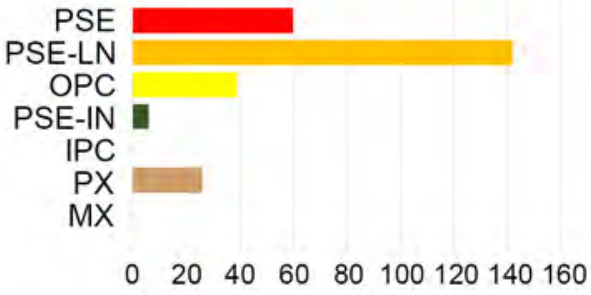

d

Number of periclinal cell divisitons

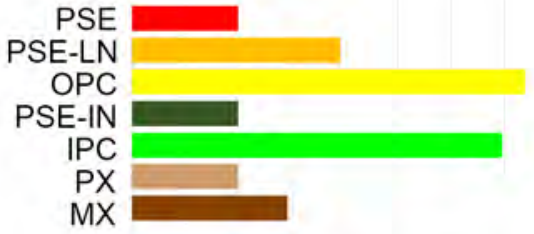

$\begin{array}{lllll}0 & 2 & 4 & 6 & 8\end{array}$

The mean number of cells in given cell type 


\section{Extended Data Fig. 1| Quantification of periclinal cell division during procambial development.}

a, Schematic representation of root vascular tissue of Arabidopsis. Procambial cells originate from their initial cells, and periclinal cell division increases the cell files during the proliferative phase, eventually resulting in a bisymmetric vascular pattern composed of a pair of phloem poles, which are separated from central xylem axis by intervening procambium. $\mathbf{b}$, An example of mapping the position of periclinal cell divisions from the initial cells. From each position within the root vascular tissue (arrows), an optical cross-section image is constructed, and cells were segmented using CellSet. c, The number of periclinal cell divisions in each cell position (273 division events from 13 independent roots). d, The mean cell number in each category during procambial development. The number of events per cell in each group was calculated by diving the number of events by the mean cell number of each group during development (See Supplementary Information). 

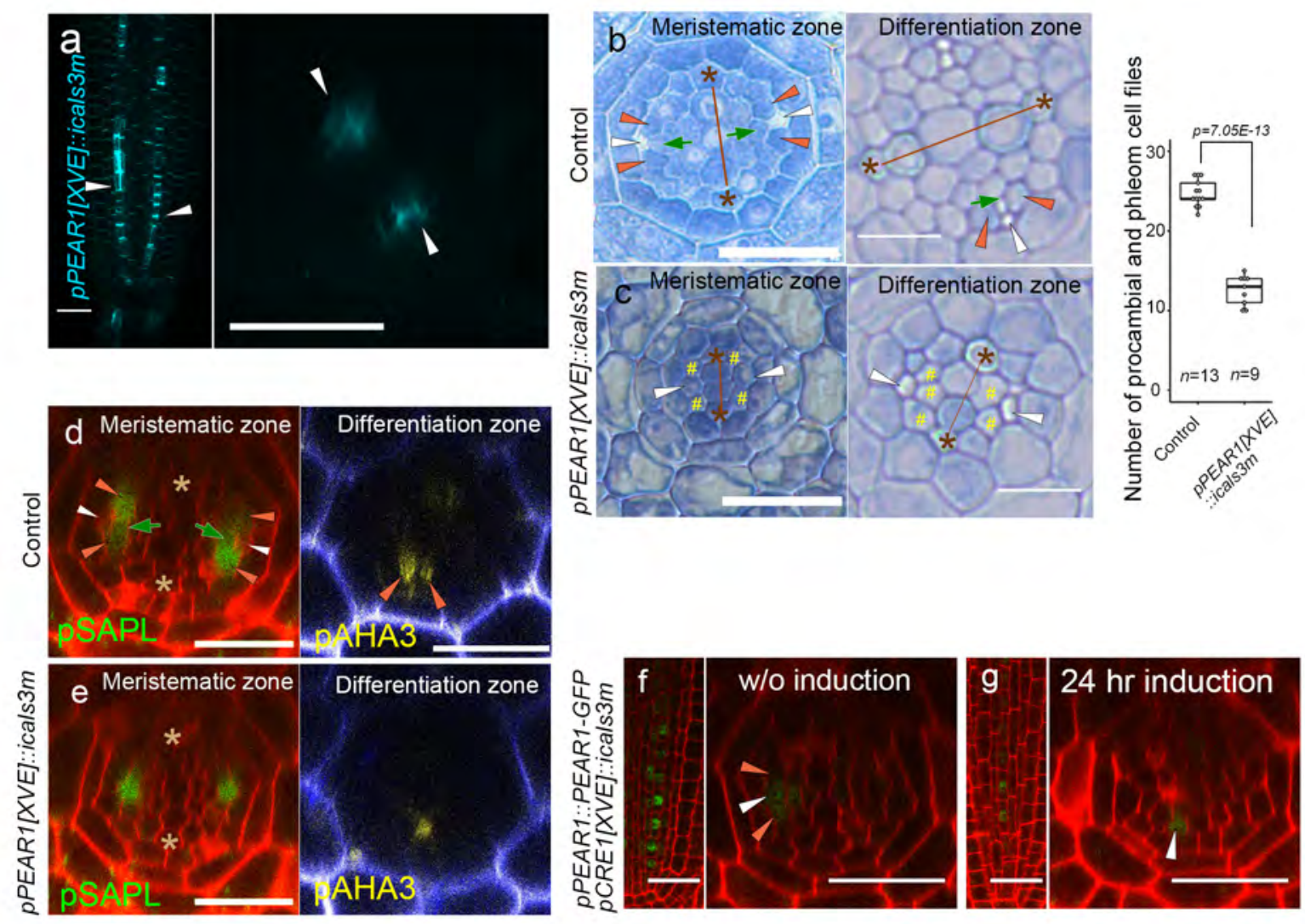
Extended Data Fig. 2| Inhibition of symplastic connection in early PSE results in the reduction of vascular cell number and in PSE-specific PEAR1-GFP localization.

a, Aniline blue stained primary root of pPEAR1[XVE]::icals $3 m$ after 24 hours of induction. Callose deposition occurs superficially in PSE cells (arrowheads, $n=10$ ). b-c, The vascular tissue of pPEAR1 [XVE] ::icals $3 m$ root, in non-induced $(\mathbf{b}, n=13)$ and after three days induction $(\mathbf{c}, n=9)$. In noninduced condition, PSE cells (white arrowheads) and their neighbouring cells, composed of MSE (dark-green arrows) and two lateral companion cells (orange arrowheads), are spatially separated from the xylem axis by intervening procambium. By contrast, after three days induction of callose deposition in PSE cells, only a single SE cell file is formed in each phloem pole (c, white arrowheads), and its neighbouring cells often touch the xylem axis (c, yellow hashtags). Number of procambial and phloem cell files is significantly reduced after three days induction. Boxplot centres show median. For more information on boxplots, see Methods. $P$ value was calculated by two-sided Student's t-test. d-e, Expression of Sister of APL (SAPL, AT3G12730) and ATPase 3 (AHA3, AT5G57350) in pPEAR1[XVE] ::icals $3 m$ before (d, $n=10$ and 4, respectively) and after 24 hours of induction (e, $n=10$ and 4, respectively). $S A P L$ is expressed in CC and MSE in meristematic zone, and $A H A 3$ is expressed in differentiated CC (d). After induction, expression of these genes is restricted to a single cell file, indicating that symplastic cell communication between PSE and PSE-LN is required for the specification of PSE-neighbouring cell identity. f-g, PEAR1-GFP localization in $p C R E 1[X V E]:: i c a l s 3 m$ before $(\mathbf{f}, n=8)$ and after 24 hours of induction $(\mathbf{g}, n=7)$. PEAR1-GFP becomes specific to the PSE cell after the induction of callose deposition in whole vascular tissue, suggesting that PEAR1-GFP move in a short rage via plasmodesmata. White, orange and dark-green arrowheads indicate PSE, PSE-LN and PSE-IN, respectively. Asterisks indicate protoxylem (PX) cells. In a-g, $n$ represents independent biological samples. Scale bars, $25 \mu \mathrm{m}$. 
a

Relative expression level GATA20/S32

Dof-type transcription factor NAC-type transcription foctor At2g37590 (PEAR1/DOF2.4) At1g65910 (ANAC028) At5g02460 (PEAR2/DOF5.1) At1g54330 (ANAC020) At3g45610 (DOF6/DOF3.2) GATA-type transcription factor At5g62940 (HCA2/DOF5.6) At4g36620 (GATA19)

MADS-box transcription factor At2g18380 (GATA20/S32) At2g42830 (SHP2/AGL5) b

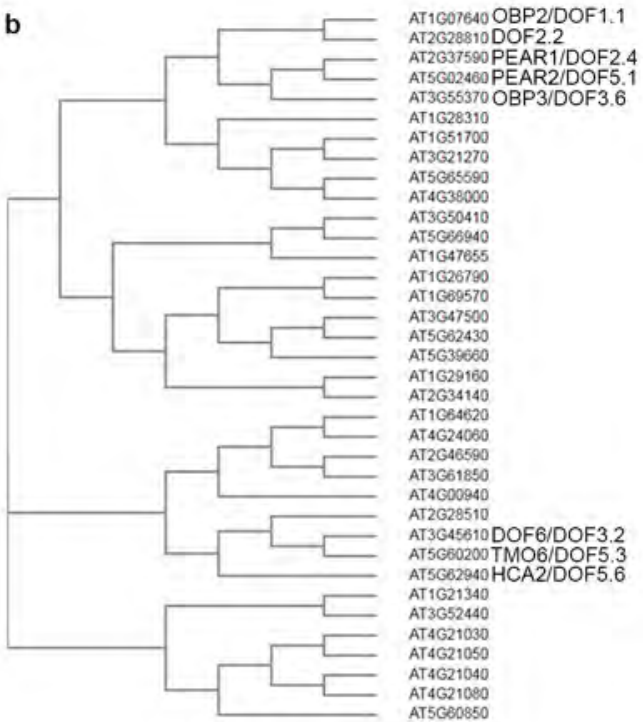

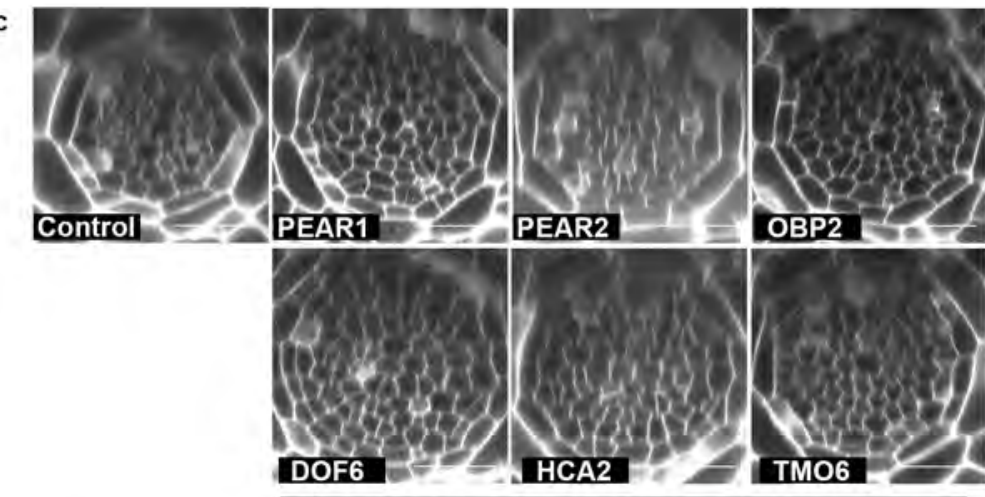

PCRE1[XVE]::DOF (24 hr induction)



$\overline{\text { PCRE1[XVE]::DOF (24 hr induction) }}$
。
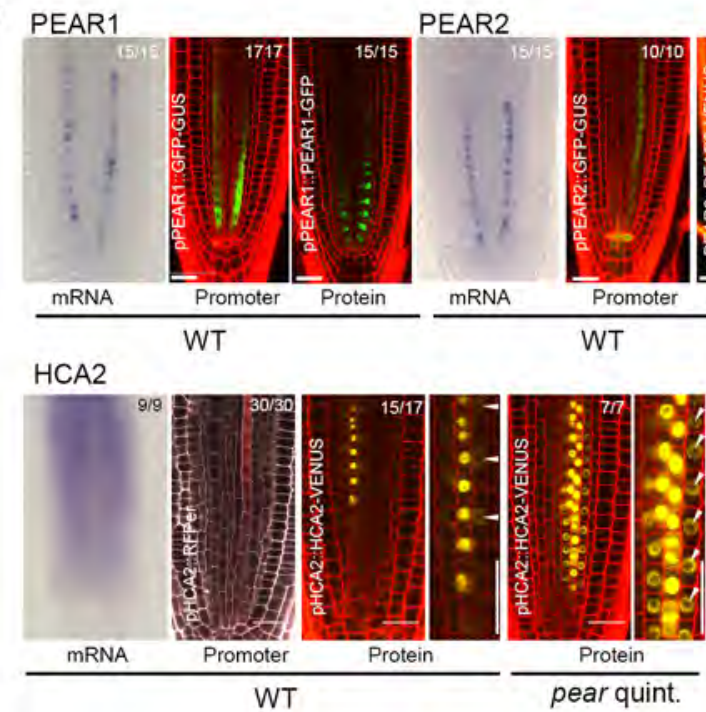

PEAR2

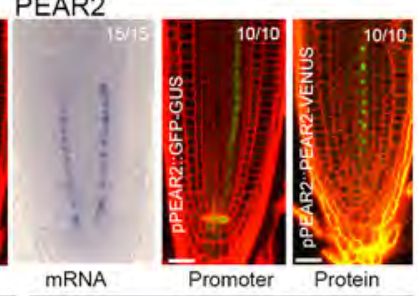

DOF6

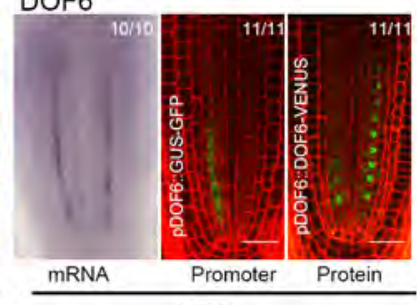

TMO6

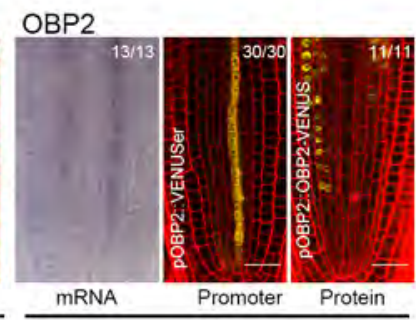

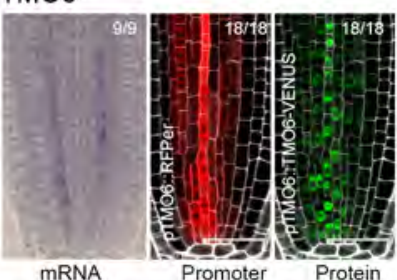

mRNA

Promoter

Protein
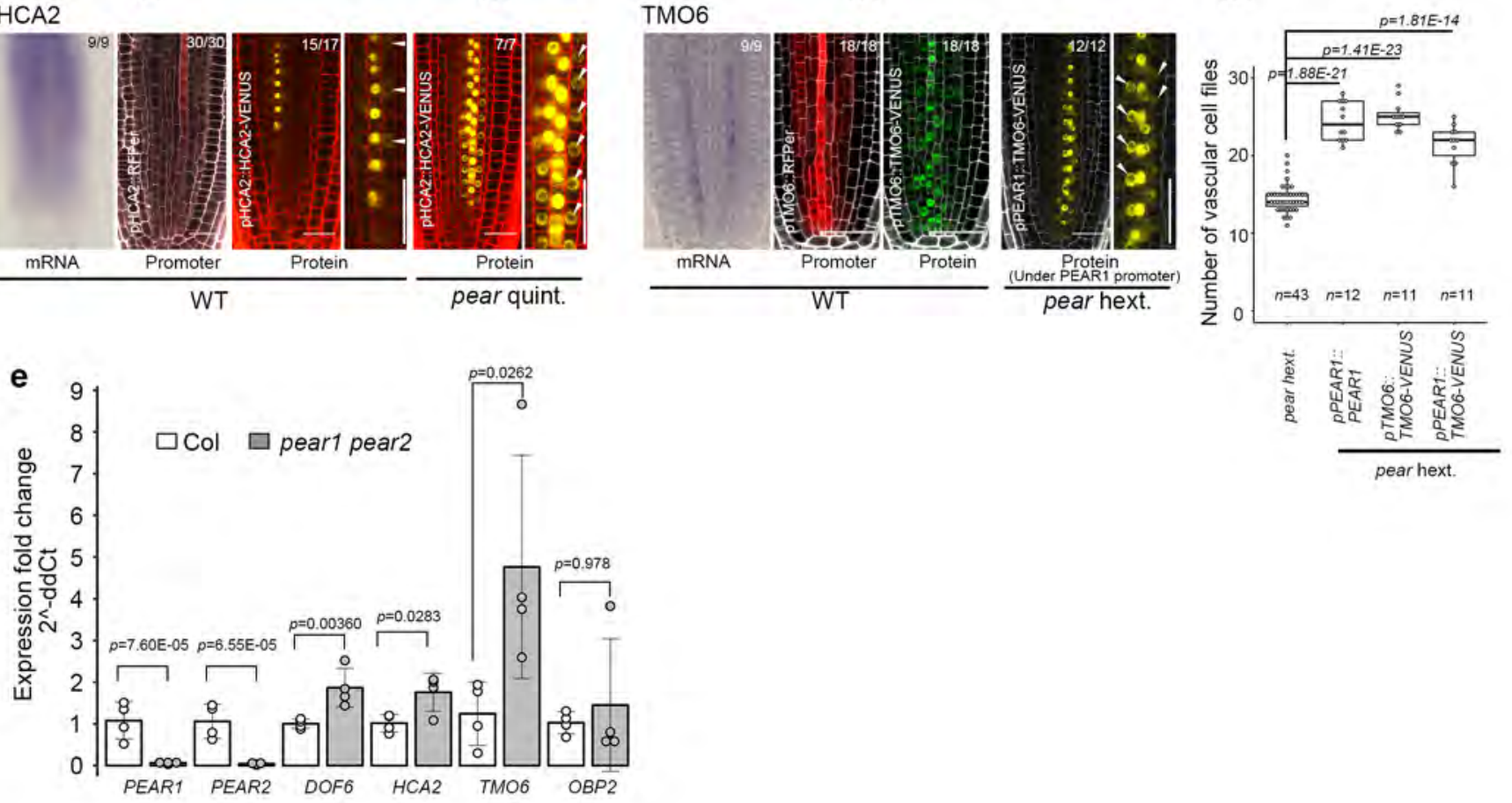


\section{Extended Data Fig. 3| Identification of PEAR genes.}

a, In silico analysis of the early phloem abundant transcription factors. Nine transcription factors are shown to be expressed abundantly in the early phloem cell (S32 fraction), containing four types of transcription factors, including DOF-type, MADS-box, NAC-type and GATA-type transcription factors. b, A phylogenetic tree of 36 Arabidopsis DOF transcription factors is drawn using Clustal Omega (https://www.ebi.ac.uk/Tools/msa/clustalo/). c, Overexpression of PEAR genes, including PEAR1, PEAR2, OBP2, DOF6, HCA2 and TMO6, under the CRE1 inducible promoter enhances periclinal cell division in the vascular tissue. $n$ represents independent biological samples. Bar graphs represent mean. Error bars are s.d. Dots, individual data points. $P$ values were calculated by two-sided Student's t-test. d, Expression of six PEAR genes, including PEAR1, PEAR2, DOF6, TMO6, OBP2 and $H C A 2$, show similar expression patterns to PEAR1, in which both mRNA and transcriptional fusion reporter exhibit PSE-specific pattern with a broad protein localization. HCA2 translational fusion in wild-type background exhibits weak but detectable signal in PSE-neighbouring cells (arrows), and its expression level is enhanced in the pear quintuple mutant background. Though TMO6 mRNA is highly specific to PSE cells, its transcriptional fusion reporter shows a broad but PSE abundant expression pattern with a broad TMO6 protein localization. Mobility of TMO6 protein is more evident when TMO6-VENUS is expressed under PSE-specific PEAR1 promoter (pPEAR1::TMO6-VENUS) in pear hextuple. Number in each panel indicates samples with similar results of the total independent biological samples analysed. Boxplot centres show median. $P$ value calculated by two-sided Student's t-test. Dots, individual data points. e, A quantitative analysis of PEAR transcripts in pear 1 pear 2 double mutant background. Note that the level of transcripts of three PEAR genes, including TMO6, DOF6 and HCA2, is elevated in pear1 pear2 background, suggesting that a compensation mechanism would mask the effect of pearl pear2 loss of function (also see Supplemental Information). Bar graphs represent mean. Error bars are s.d. Dots, individual data points. $P$ values were calculated by two-sided Student's t-test. Scale bars, $25 \mu \mathrm{m}$. 

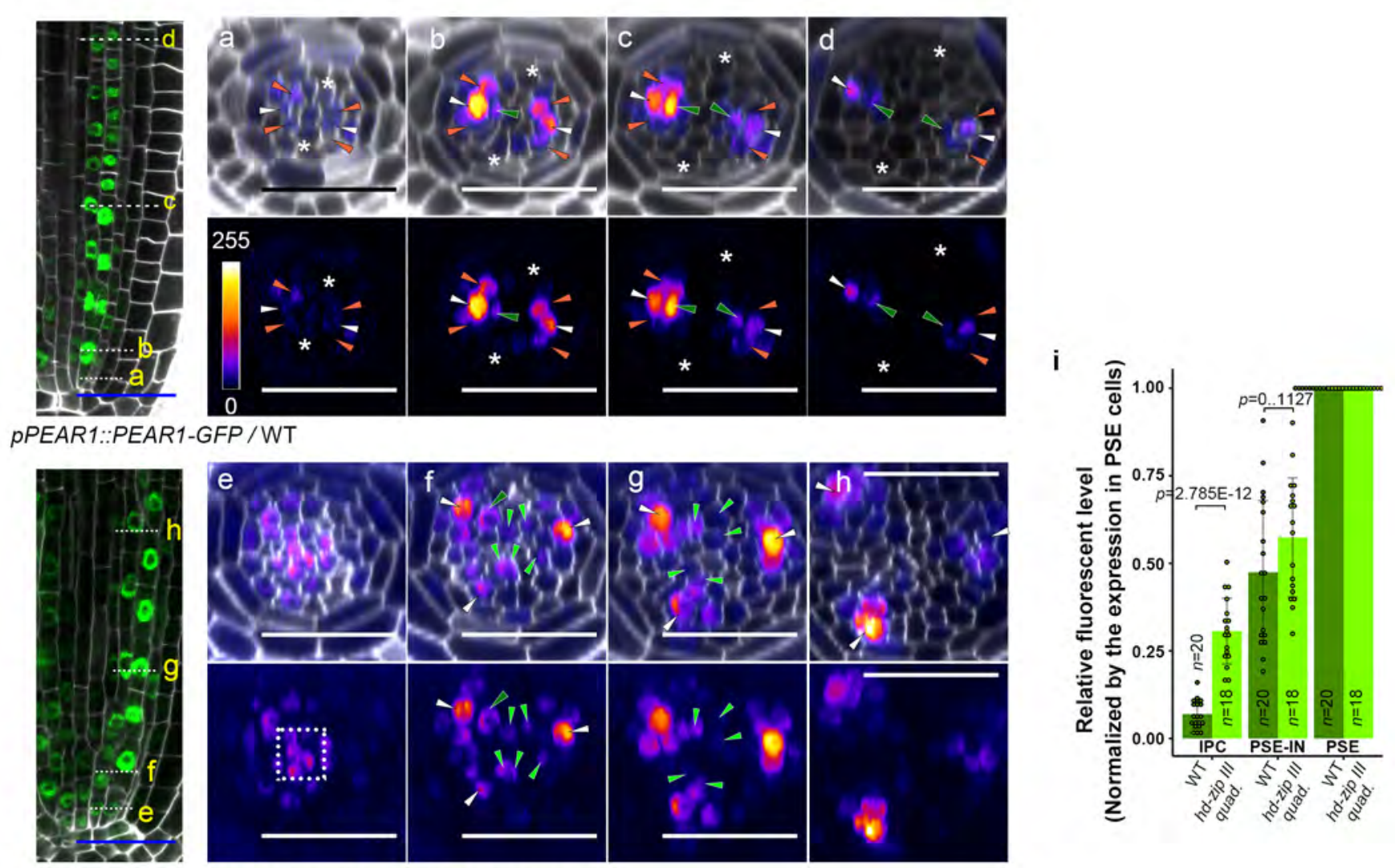

pPEAR1::PEAR1-GFP / hd-zip III quad. (phb phv cna athb8) 


\section{Extended Data Fig. 4| PEAR1-GFP localization during procambial development.}

a-d, PEAR1-GFP localization in wild-type background ( $n=19$, independent biological samples). The position of each optical section is indicated in the left panel showing the longitudinal section. At the position of the vascular initial cells, weak PEAR1-GFP signal is observed in PSE and neighbouring procambial cells but not in the xylem cells (a). During an early stage of the proliferative phase, the highest PEAR1-GFP signal is detected in the PSE, and substantial level of PEAR1-GFP signal is observed in PSE neighbouring cells, PSE-LN and PSE-IN (b, c) and its expression is maintained by the end of proliferation stage (d), indicating that the expression pattern of PEAR1-GFP is correlated with the domain having high proliferative activity, except for PSE-IN where almost no periclinal cell division is detected (see Fig. 1b). e-h, PEAR1-GFP localization in hd-zip III quadruple ( $p h b$ phv cna athb8) mutant background ( $n=17$, independent biological samples). The position of each optical section is indicated in left panel showing the longitudinal section. Broad localization of PEAR1-GFP is detected at the level of vascular initials. Central domain is highlighted with a dotted square (e). At the early stage of proliferative stage, fluorescent signal is detected in IPC cells (light-green arrowheads), as well as PSE and its neighbouring cells (f, g), and gradually becomes specific to PSE and its neighbours (h). i, Quantification of PEAR1-GFP signal in each cell type. Fluorescent intensity of PEAR1-GFP in IPC and PSE-IN cells during proliferative phase (b-c in wild-type, $\mathbf{f - g}$ in $h d$-zip III quadruple, respectively) was measured and normalized to the fluorescent intensity in PSE cells, confirming a broad distribution of PEAR1-GFP in $h d$-zip III quadruple. $n$ represents individual measurements across 5 (wild type) or 4 (hd-zip III quadruple) independent biological samples, respectively. Bar graphs represent mean. Error bars are s.d. Dots, individual data points. $P$ values were calculated by two-sided Student's t-test. White, orange and dark-green and light-green arrowheads indicate PSE, PSE-LN, PSE-IN and IPC respectively. Scale bars represent $25 \mu \mathrm{m}$. 
a

GT8483 (Ler)
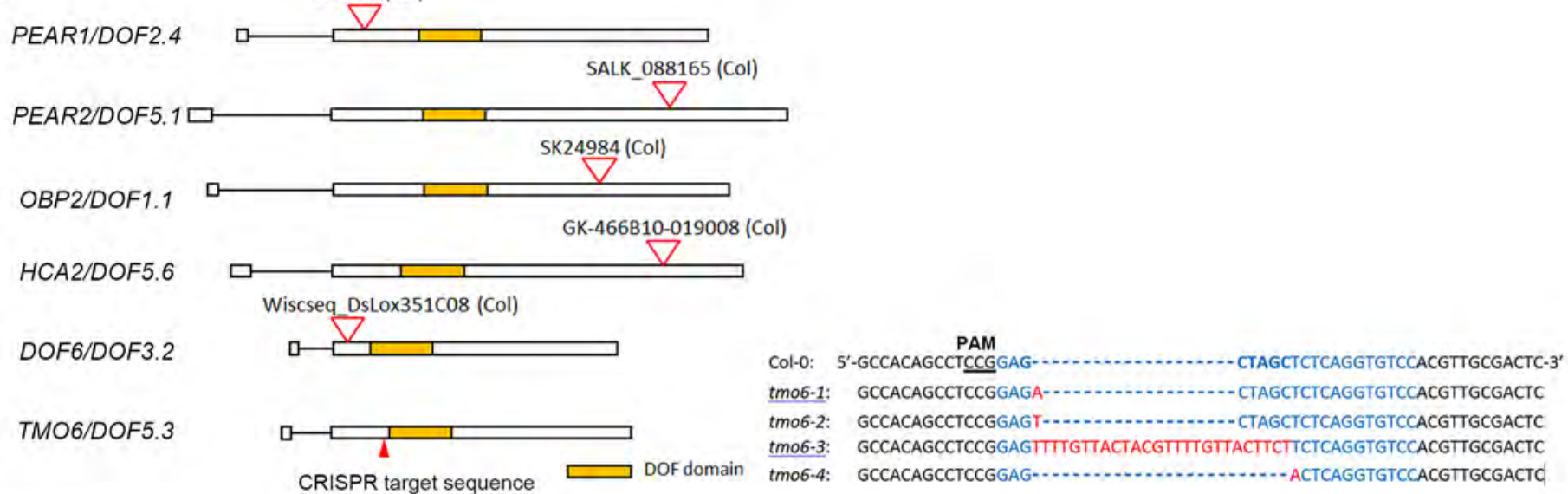

b
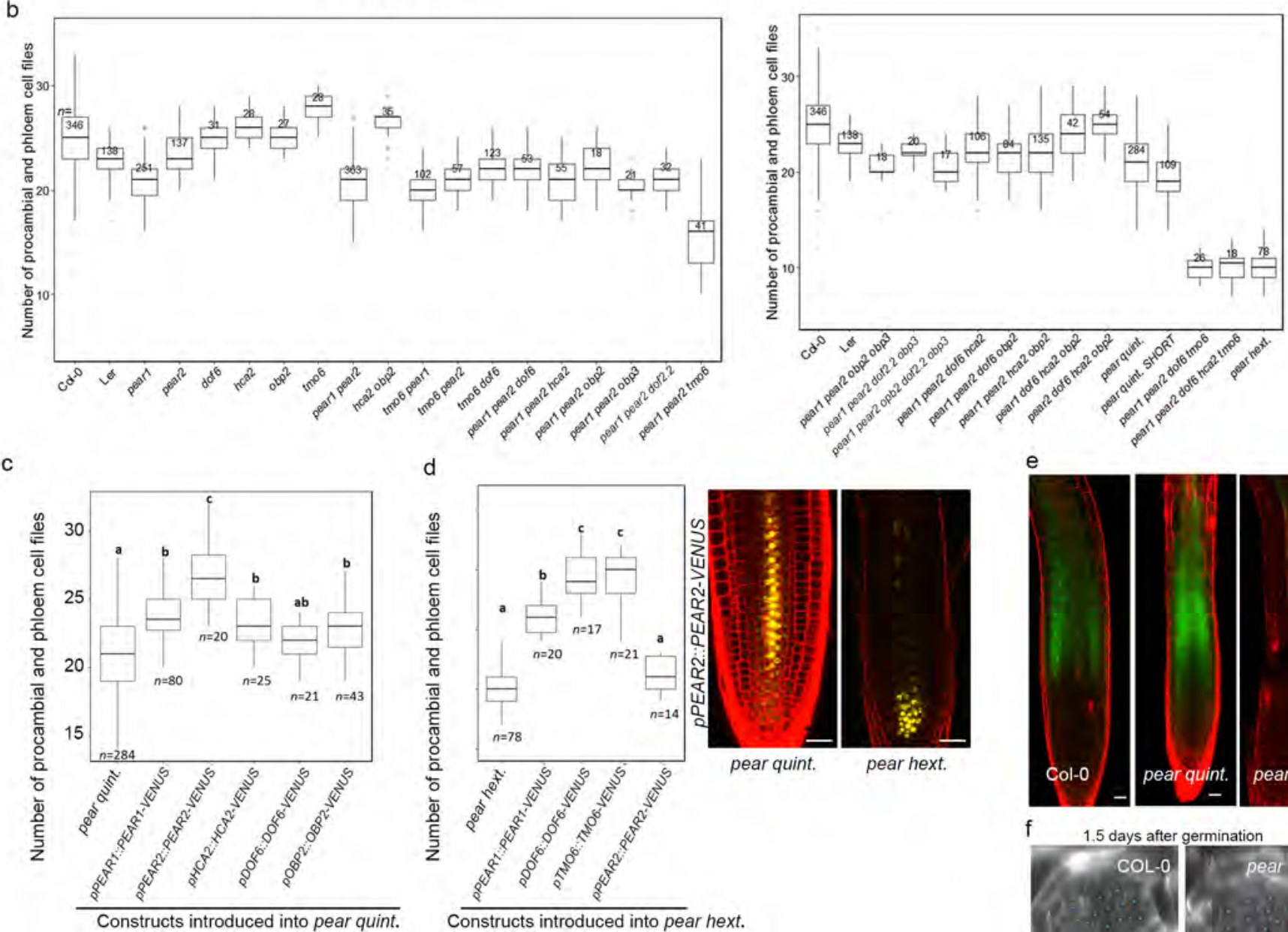

d
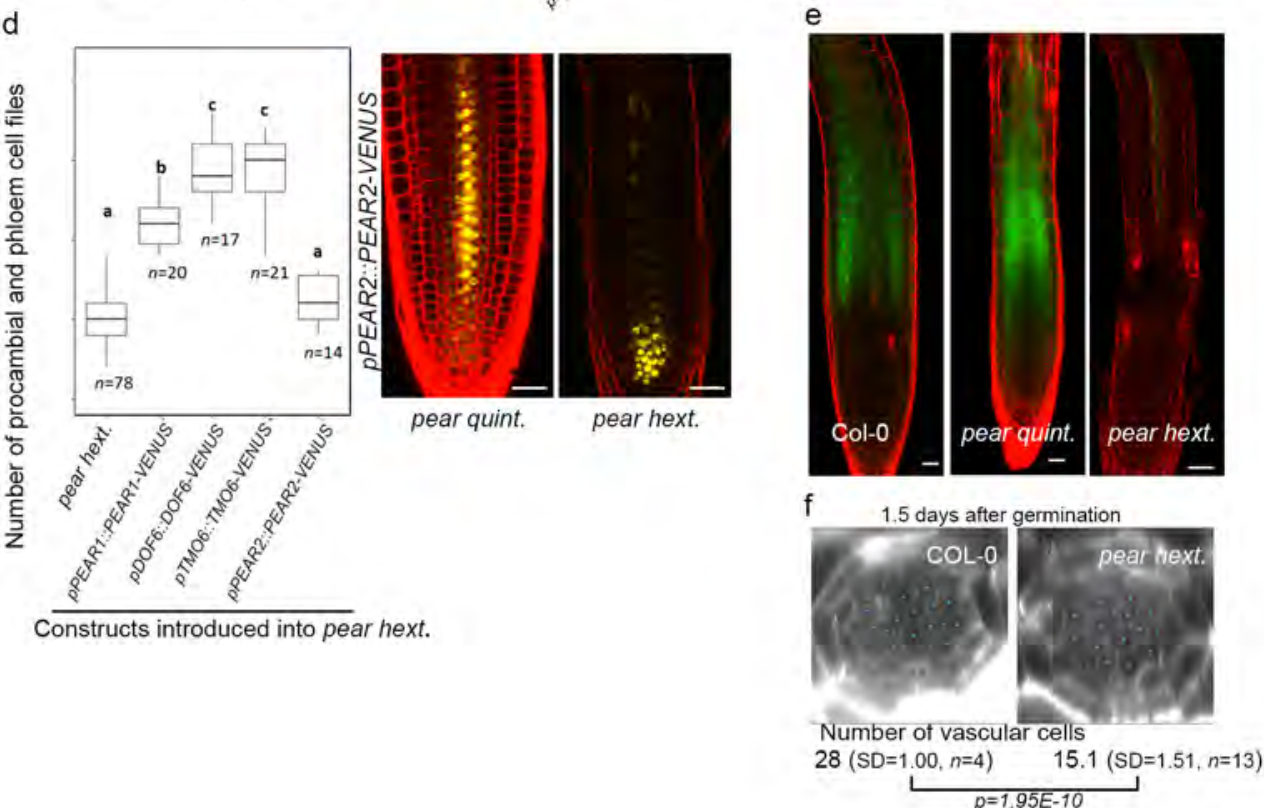


\section{Extended Data Fig. 5| Loss of function of PEAR genes.}

a, Organization of PEAR genes and CRISPR/Cas9-induced mutation in TMO6 locus. Deletions are denoted by dashes; insertions and a replacement are indicated by red letters. b, Quantification of phloem and procambium cell files in lower (left) and higher (right) order pear combinatorial mutants. Tukey's HSD test is provided for all samples in Supplementary Table 3. c, pear quintuple mutant phenotype is suppressed by introduction of fluorescent-tagged PEAR proteins expressed under their native promoters. $\mathbf{d}$, pear hextuple mutant phenotype is significantly suppressed by the introduction of PEAR1, DOF6 and TMO6 construct, but not PEAR2. In the pear hextuple background, PEAR2 expression is highly reduced in the vascular tissue. e, Phloem unloading assay in wild type, pear quintuple (with shortest roots) and pear hextuple ( $n=15,8$ and 15, respectively). Fluorescent CFDA dye is loaded on the cotyledon and imaged two hours after application (see Methods). Phloem transport and unloading is not changed in the shortest roots of the pear quintuple mutant strongly affected in the radial growth. pear hextuple shows defects in phloem transport. f, Phenotype of pear hextuple mutant at the early developmental stage (1.5 days after germination). The cell number in vascular tissue of pear hextuple is significantly reduced before the onset of phloem PSE differentiation and activation of the phloem transport (see Supplementary Information). $P$ value was calculated by two-sided Student's t-test. In b-f, $n$ represents independent biological samples. In b-d, statistically significant differences between groups were tested using Tukey's HSD test $\mathrm{p}<0.05$. For individual $P$ values, see Supplementary Table 3. Boxplot centres show median. For more information on boxplots, see Methods. Scale bars represent $25 \mu \mathrm{m}$. 

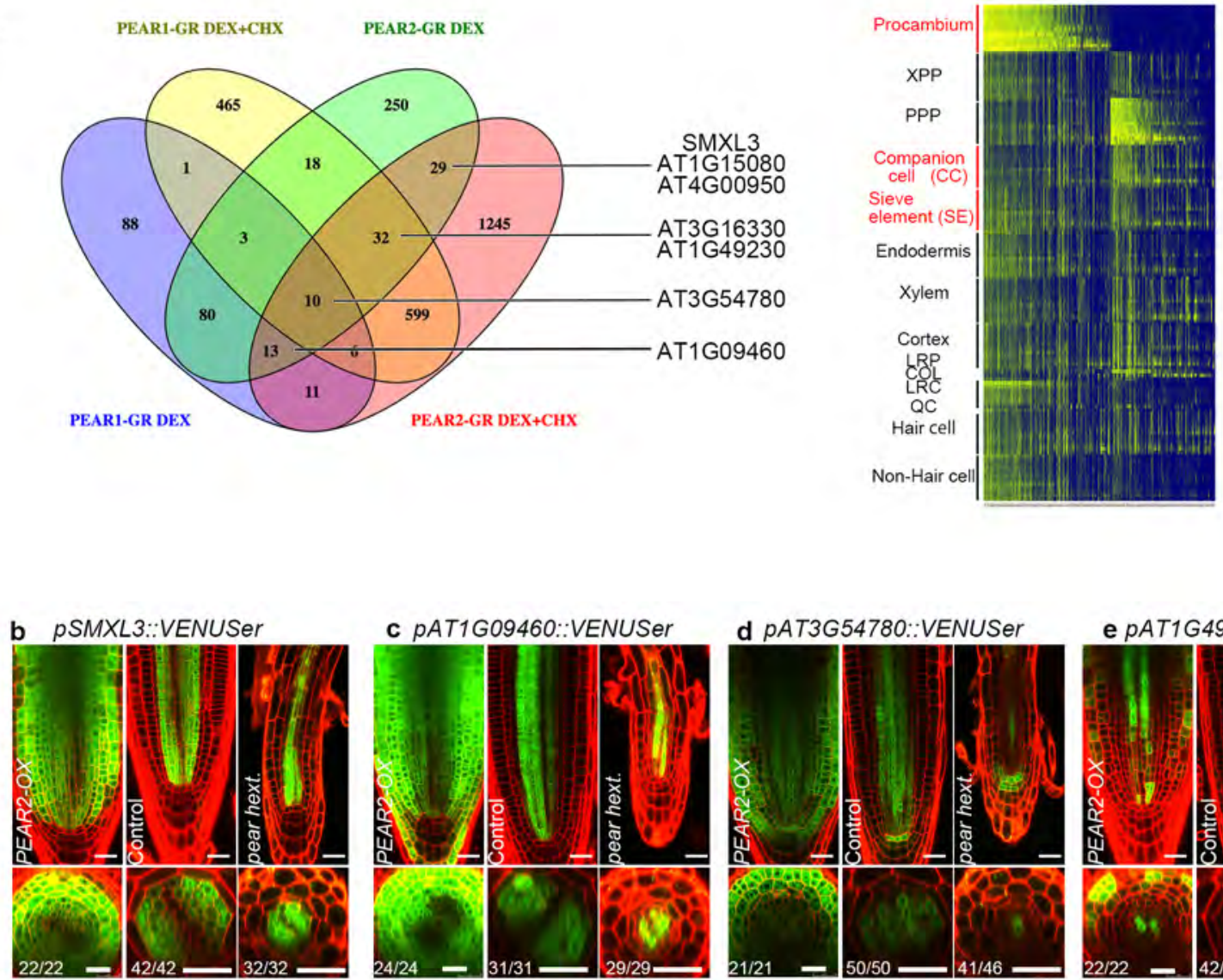

c pAT1G09460::VENUSer
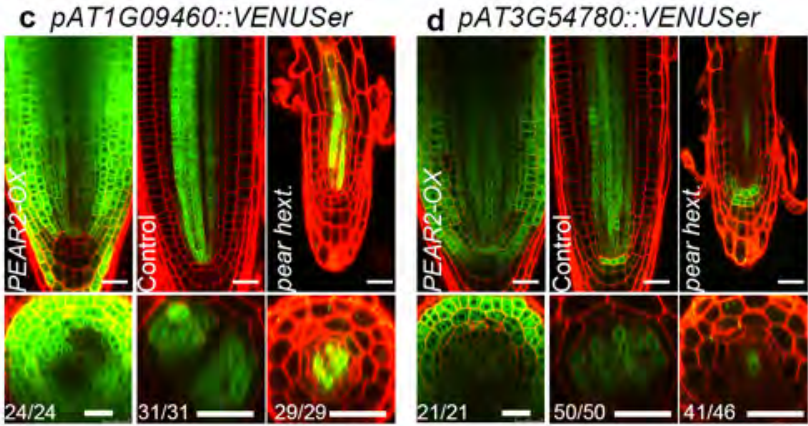

e pAT1G49230::VENUSer

f PAT1G15080::VENUSer

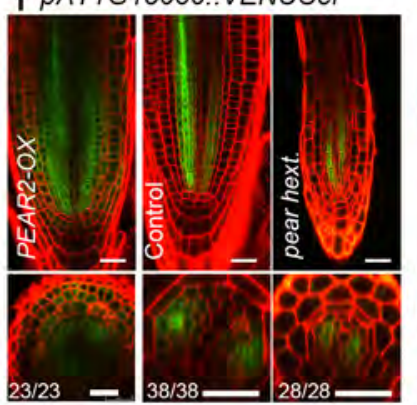

g pAT3G16330::VENUSer

h PAT4G00950::VENUSer
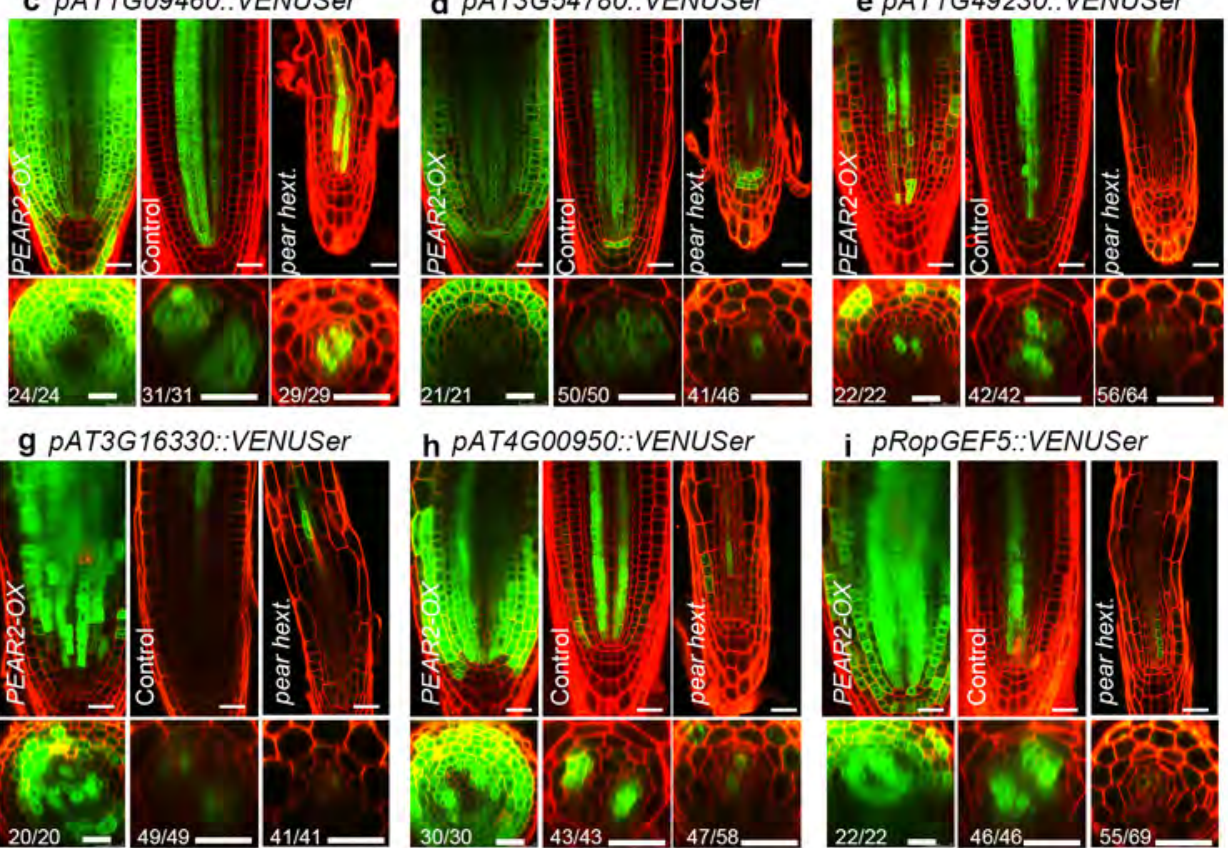

i pRopGEF5::VENUSer

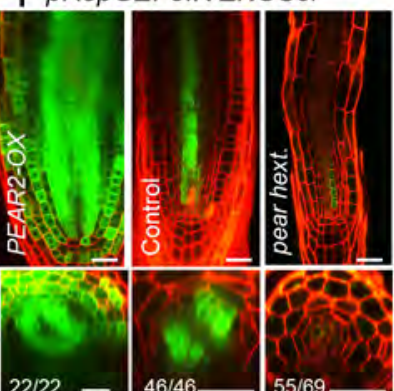

j


AT1G09460 ROP-GEF5 AT3G16330 AT3G54780 SMXL3 


\section{Extended Data Fig. 6| Identification of genes acting downstream of PEAR.}

a, Venn diagram showing the genes upregulated by overexpression of PEAR1 or PEAR2 with and without cycloheximide (chx). The analysis revealed 212 and 435 upregulated genes, in the respective experiments. Heatmap showing the predicted spatiotemporal expression patterns of all genes induced by PEAR1 or PEAR2. b-i, Expression patterns of eight selected genes responding to PEAR2 overexpression. In control conditions, all genes exhibit a broad expression pattern, in which five of them are transcribed both in phloem and procambial cells (b-d and i), and the rest of them are in PSE and its surrounding cells where PEAR proteins are accumulated abundantly (e-h). Whereas expression of SMXL3, AT1G09460 and AT1G15080 are maintained even in pear hextuple (b, c, f), the expression level of five genes are attenuated (d, e, g-i). Number in each panel indicates samples with similar results of the total independent biological samples analysed. $\mathbf{j}$, Number of vascular cells after 3-days induction of overexpression of each PEAR downstream gene. In each case, several lines were analysed in parallel for a phenotypic change. Only $S M X L 3$ overexpression can increase the vascular cell number (confirmed in three independent lines). Boxplot centres show median. For more information on boxplots, see Methods. $n$ represents independent biological samples. Dots, individual data points. $P$ value was calculated by two-sided Student's t-test. Scale bars represent $25 \mu \mathrm{m}$. 

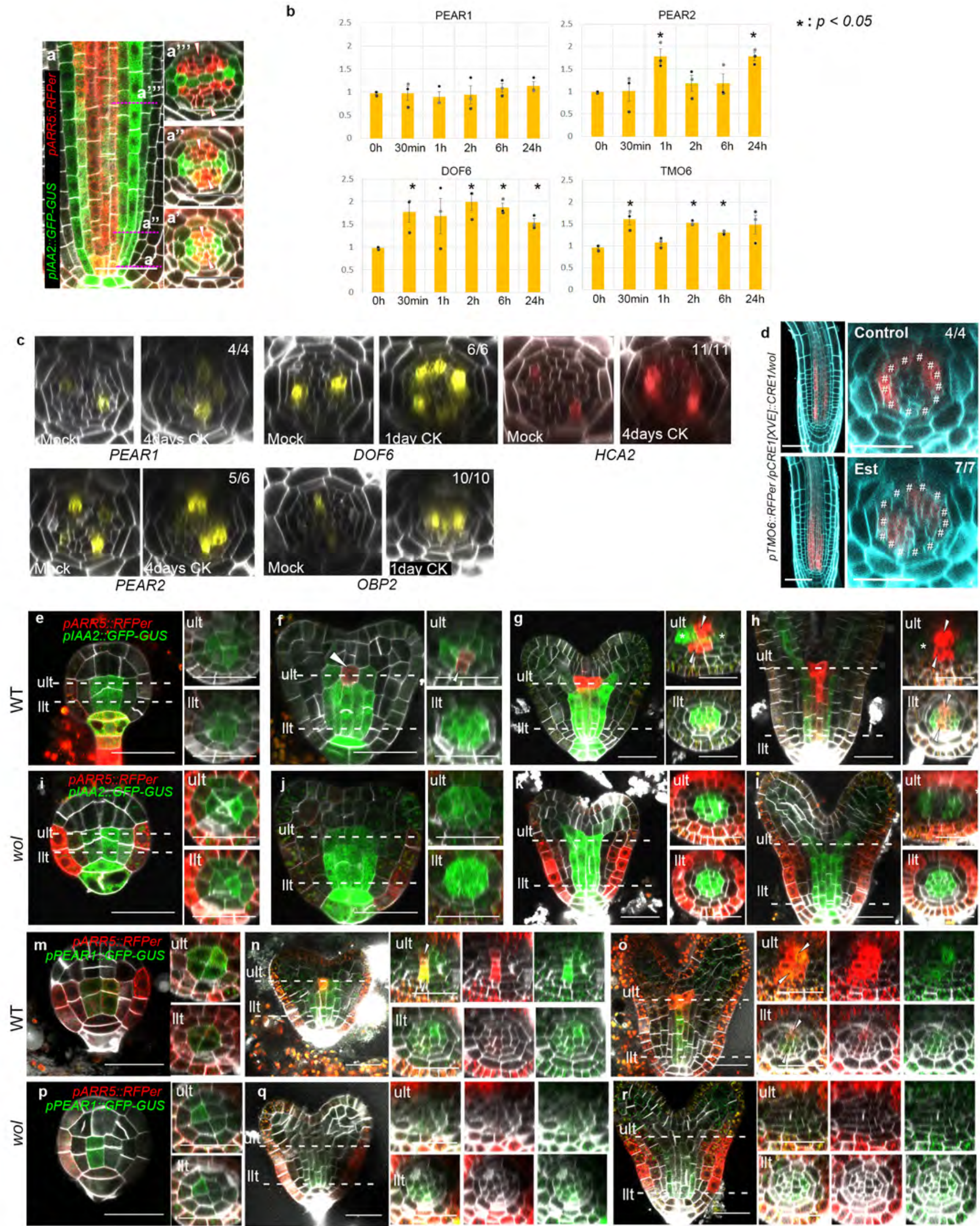


\section{Extended Data Fig. 7| Cytokinin controls PEAR expression.}

a, Expression of auxin ( Auxin response is restricted to the xylem cells at initial stage (a') and maintained during development (a", a"). High cytokinin response is activated initially and maintained in PSE and its neighbouring cell (a', aa'), and later becomes concentrated into the intervening procambial cells flanking xylem cells (a"'). b, Exogenous cytokinin application rapidly promotes the transcript level of PEAR genes, including PEAR2, DOF6, and TMO6. Asterisks indicate significant $(\mathrm{p}<0.05)$ upregulation as determined by a two-sided t-test on three biological replicates. Bar graphs represent mean. Error bars are s.e.m. For individual $P$ values, see Supplementary Table 3. c, Sustained cytokinin application leads to ectopic transcription of $P E A R$ genes. The optical cross section images are obtained after 4 days (for PEAR1, PEAR2 and HCA2) or 1-day (DOF6 and OBP2) treatment of $1 \mu \mathrm{M}$ of BA. d, Conditional induction of CRE1 expression restores TMO6 transcription in wol root. In the absence of cytokinin response, TMO6 transcription is increased in pericycle and attenuated in the vascular tissue (Control, hashtags), and is restored in the vascular tissue after CRE1 induction (Est). e-l, Expression pattern of auxin (pIAA2::GFP-GUS) and cytokinin (pARR5::RFPer) response reporters during embryogenesis in wild type (e-h, $n=29,13,13,11$, respectively) and wol (i-l, $n=12,8,6$, respectively). At globular stage, auxin response is activated among provascular cells both in wild type (e) and in wol (i). At early heart stage, cytokinin response is activated in cells positioned below shoot apical meristem (f, ult, arrowheads), and the stripe of cytokinin response domain is formed by mid heart stage (g, ult, arrowheads), simultaneously auxin response becomes concentrated in the cells proximal to the cotyledon (g, ult, asterisks), resulting in the bisymmetric hormonal response pattern. During the torpedo stage, cytokinin response domain reaches to llt (h, llt, arrowheads). In wol embryos, activation of cytokinin response in vascular tissue does not occur and a radial auxin response pattern is maintained (i-l). m-r, Expression of $A R R 5$ and PEAR 1 during embryogenesis. In the wild-type embryo (m-o, $n=17$, 25,10 , respectively), $P E A R 1$ is broadly transcribed among provascular cells both in ult and 1lt with radial symmetric pattern at the globular stage $(\mathbf{m})$. At the heart stage, PEAR1 transcription is enhanced in ult cells underneath the shoot apical meristem, which correlated with the activation of cytokinin response in this domain (n, arrowheads), and expression of both ARR5 and PEARI extends rootward and reaches to llt, becoming more concentrated within the cell files where phloem is specified postembryonically (o, arrowheads). In wol embryos (p-r, $n=19,13,13$, respectively), PEAR1 transcription is initiated among provascular cells at the globular embryo stage (p) similar to wild type (m), but neither cytokinin response nor PEARl transcription occurs in ult at the heart stage (q), and PEARI expression is gradually attenuated by the torpedo stage (r). ult and llt represent upper- and lower tier, respectively. In a, e-r, $n$ represents independent biological samples. In $\mathbf{c}$ and $\mathbf{d}$, number in each panel indicates samples with similar results of the total independent biological samples analysed. Scale bars represent $25 \mu \mathrm{m}$. 




\section{Extended Data Fig. 8| HD-ZIP III restrict periclinal cell divisions during procambial development.}

a-c, Periclinal cell divisions in the cells non-adjacent to pericycle, including PSE-IN (a), IPC (b) and xylem cell (c), occurs in $h d$-zip III quadruple (phb phv cna athb8). $n=8$. d-e, The number of periclinal cell divisions in cells adjacent (d) or non-adjacent to pericycle (e) in wild type, pear and hd-zip III combinatorial mutants. In the analysis of pear quintuple and pear hd-zip III nonuple, a population of slowly elongating roots is selected as described in Fig. 2f. Boxplot centres show median. Statistically significant differences between groups were tested using Tukey's HSD test $\mathrm{p}<0.05$. For individual $P$ values, see Supplementary Table 3. f-i, Localization of PHB-GFP (f, $n=12)$, CNA-GFP (g, $n=10)$, REVGFP $(\mathbf{h}, n=7)$ and ATHB8-GFP $(\mathbf{i}, n=5)$. $\mathbf{j}-\mathbf{k}$, Protein localization of PHB-GFP $(\mathbf{j}, n=7)$ and CNA-GFP $(\mathbf{k}, n=4)$ during procambial development. In the initial cells $\left(\mathbf{j}^{\prime}, \mathbf{k}^{\prime}\right)$, both proteins are localized in metaxylem cells but not in PSE (white arrowheads). During the proliferation stage, PSE-IN (green arrowheads), which is produced by periclinal cell division in PSE, acquires the expression of both PHB-GFP (j", $\mathbf{j} "$ ") and CNA (k", k"'). l-o, Overexpression of PEAR1-VENUS under the CRE1 inducible promoter in wild type (l, $\mathbf{n}, n=5,3$, respectively) and heterozygous $p h b$ - $1 d$ background ( $p h b$ $1 d /+, \mathbf{m}, \mathbf{o}, n=5,5$, respectively). After 18 hours of induction, PEAR1-VENUS signal is detected in both backgrounds $(\mathbf{l}, \mathbf{m})$, however, enhanced periclinal divisions are only observed in wild type (I), and not in $p h b-1 d(\mathbf{m})$. Longer induction of PEARl overexpression induces divisions even in $p h b-1 d$ $(\mathbf{n}, \mathbf{o})$. p-r, $p P E A R 1-P E A R 1-G F P$ expression is reduced in heterozygous $p h b-1 d /+$ background. Most of phb-1d heterozygotes exhibit a single PEAR1-GFP expressing pole $(\mathbf{q}, 72 \% n=11)$, and the expression of PEAR1-GFP is almost completely abolished in some roots of $p h b-1 d(\mathbf{r}, 18 \% n=11)$. s$\mathbf{t}$, The expression of pPEAR $1: \because$ GFPer in wild type $(\mathbf{s}, n=10)$ and $\operatorname{shr}-2(\mathbf{t}, n=9)$. The fluorescent signal is below the limit of detection in shr-2. $\mathbf{u}-\mathbf{w}$, Expression of pPEAR1::GFP-GUS in WT $(\mathbf{u}, n=19)$ and $h d$-zip III quadruple mutant $(\mathbf{w}, n=11)$. In $\mathbf{a}-\mathbf{w}, n$ represents independent biological samples. White, orange and dark-green arrowheads indicate PSE, PSE-LN, PSE-IN respectively. Scale bars represent $25 \mu \mathrm{m}$. 

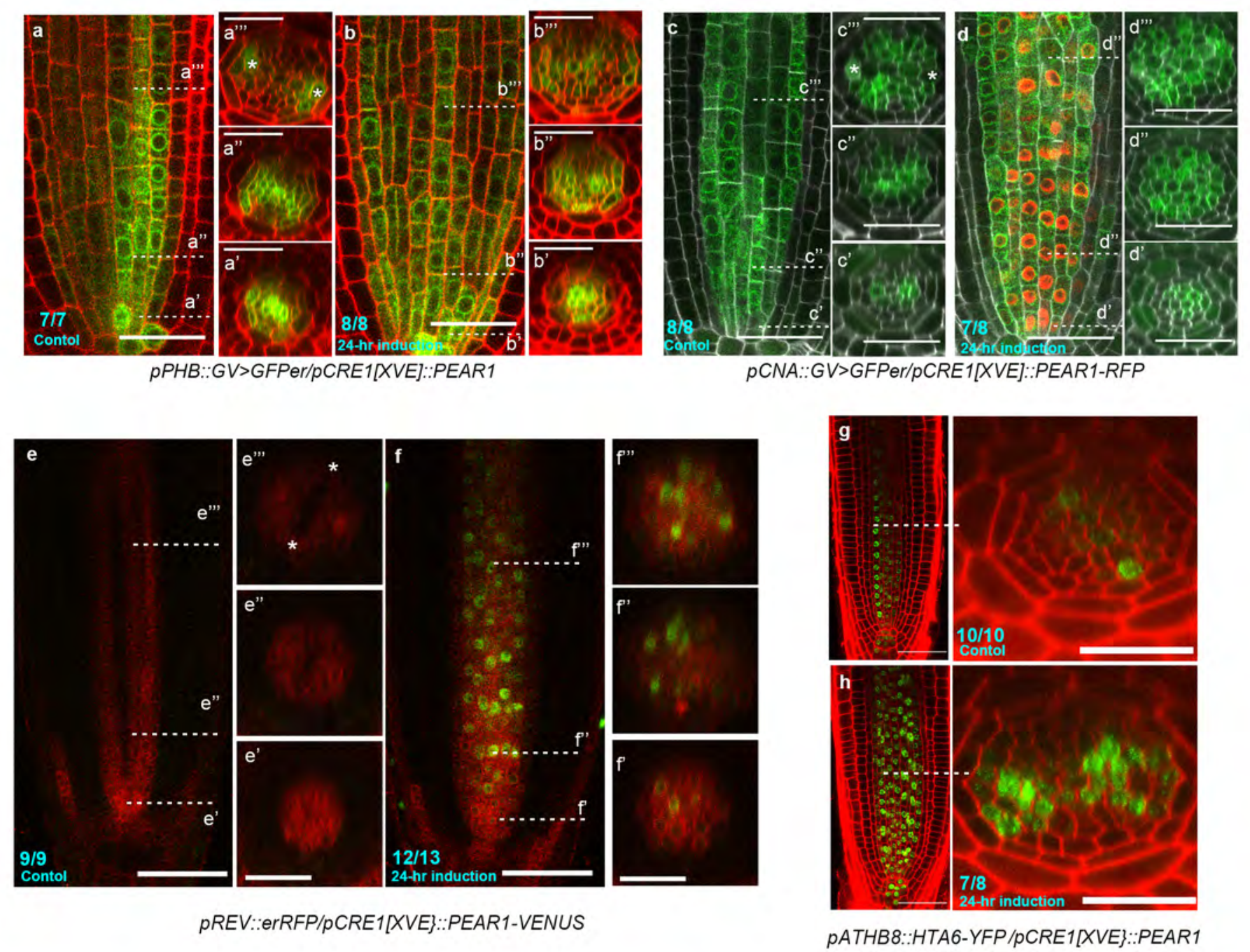


\section{Extended Data Fig. 9| Overexpression of PEAR1 enhances the transcription of HD-ZIP III.}

The transcription patterns of four HD-ZIP III, including $P H B(\mathbf{a}, \mathbf{b}), C N A(\mathbf{c}, \mathbf{d}), R E V(\mathbf{e}, \mathbf{f})$ and $A T H B 8$ $(\mathbf{g}, \mathbf{h})$ are visualized using their transcriptional fusion constructs. A longitudinal section is shown in the left panel, and the optical cross sections associated with this are shown in the right panels (the position of each section is indicated in the left panel). a-b, Transcription pattern of $P H B$ (pPHB::GV>UAS::GFPer) in $p C R E 1[X V E]: \because P E A R 1$ plant before (a) and after 24 hours of induction of PEAR1 overexpression (b). $P H B$ transcription is observed in whole vascular tissue at the initial and proliferative phase with peaks in xylem cells (a'), and its expression becomes concentrated into protoxylem cells (a" and a", asterisks indicate protoxylem cell). After the induction of PEAR1 overexpression, $P H B$ expression in the central domain of the vascular tissue is maintained at the later stage, resulting in the radially symmetric $P H B$ transcription pattern (b' and b"). c-d, Transcription of CNA ( $p C N A: \because G V>U A S: \because G F P e r)$ in $p C R E 1[X V E]: \because P E A R 1-R F P$ plant before (c) and after 24 hours of induction (d). CNA transcription is observed mainly in xylem lineage at initial cells (c'), and becomes broader in whole vascular tissue, with peaks in procambial tissue, including PSE neighbouring cells (c"), and eventually its expression is gradually reduced in PSE and metaxylem, but is maintained in procambium, PSE neighbouring cells, as well as protoxylem cells (c"'). In a similar manner to $P H B$, $C N A$ transcription in the central domain of the vascular tissue is maintained at the later stage when PEAR1-RFP is overexpressed (d"'). e-f, Transcription of REV (pREV::RFPer) in $p C R E 1[X V E]:: P E A R 1-V E N U S$ plant before (e) and after 24 hours of induction (f). REV exhibits a distinct transcriptional pattern where its expression is initially uniform in vascular tissue (e'), and highest expression is localized in PSE, while decreasing towards xylem axis ( $\mathbf{e}$ " and $\mathbf{e}$ "'). When PEAR1-VENUS is overexpressed (f), the transcription pattern of $R E V$ is also activated in the central domain of vascular tissue, resulting in the radial symmetric $R E V$ transcription pattern. g-h, The expression pattern of $p A T H B 8:: H T A 6-Y F P$ is highly specific to xylem cells (g), and its expression is enhanced after 24 hours of induction of PEAR1 overexpression with a broad expression domain (h). Number in each panel indicates samples with similar results of the total independent biological samples analysed. 


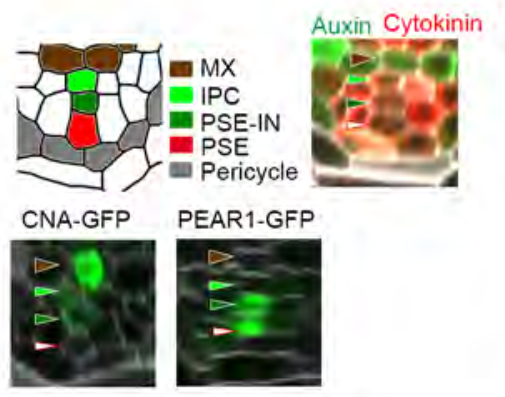

b

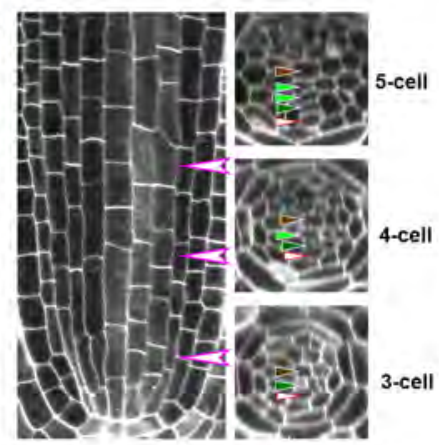

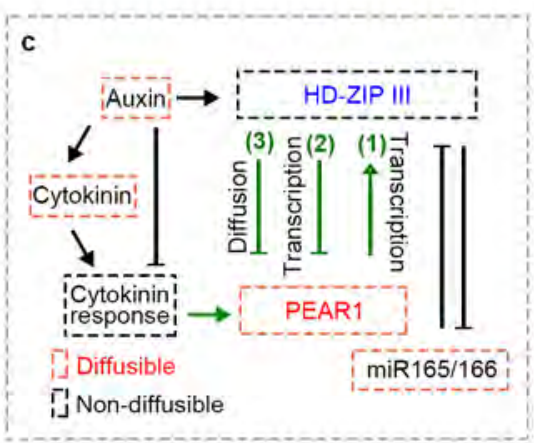

Non-diffusible d


e

CNA-GFP

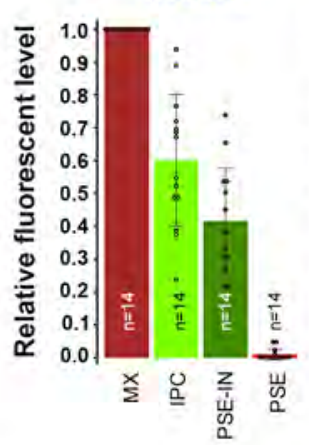

$$
\text { PEAR1-GFP }
$$
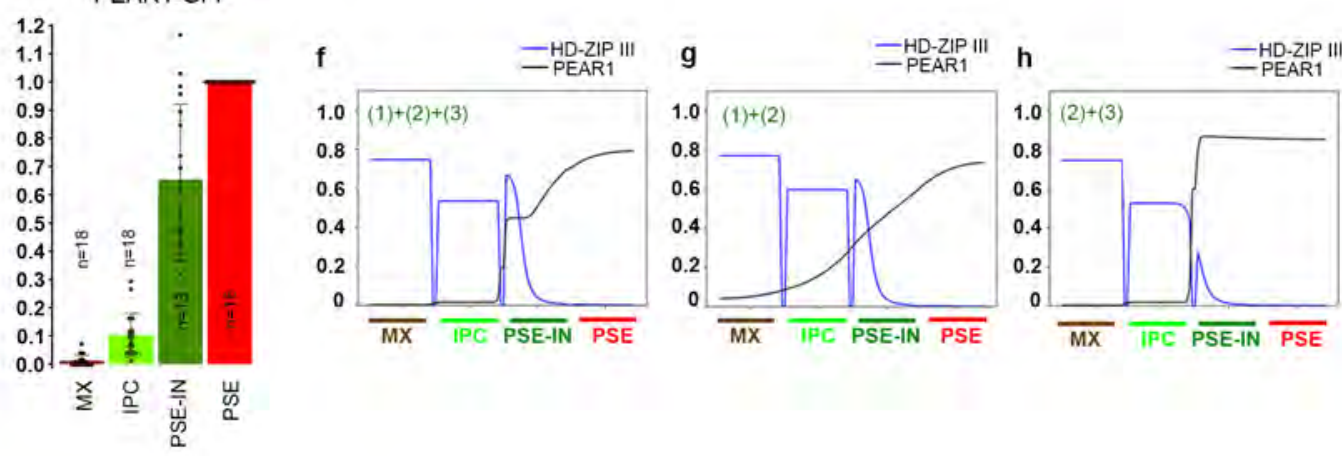

i

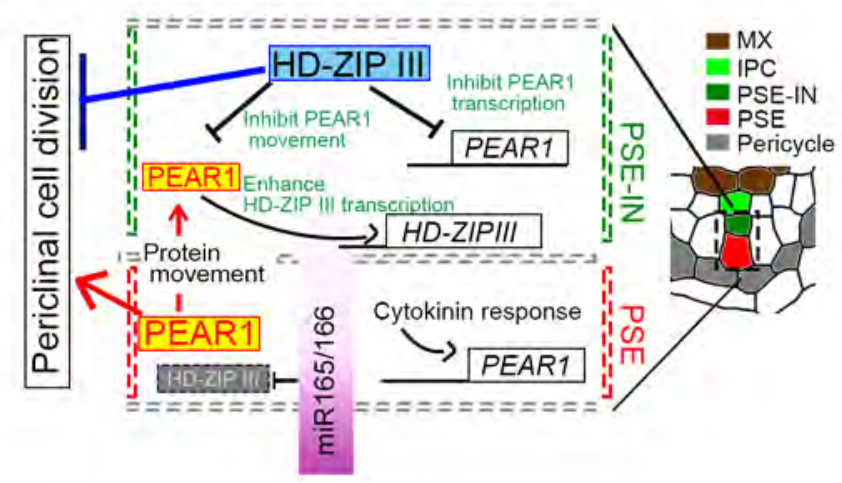


Extended Data Fig. 10| The boundary between HD-ZIP III and PEAR proteins forms within the PSE-IN.

a, Summarizing results on pattern of auxin-cytokinin (data shown in Extended Data Fig. 7a), HD-ZIP III (CNA, data shown in Extended Data Fig. 8g and k) and PEAR (PEAR1, data shown in Fig. 1d and Extended Data Fig. 4a-d) in procambium. In the simulation, we simulate the concentration of HD-ZIP III and PEAR1 along the axis between metaxylem (MX, brown arrowheads) and PSE (white arrowheads). b, Summarizing result of procambial development. Number of cells between metaxylem and PSE increases during procambial development (data shown in Extended Data Fig. 1). Therefore, the model is defined as a line in one spatial dimension representing 3, 4 or 5 cells from the centre of the xylem axis to the outer edge of the PSE cell. c, The regulatory network embedded within each cell. Regulatory interactions shown using a black line have been published previously, whilst those using a green line are described for the first time here. d, Predicted concentration gradient of all elements in 3, 4 or 5 cells (from left to right). Within different root geometries corresponding to different growth stages in Arabidopsis, both PEAR1 and HD-ZIP III are co-localized in PSE-IN, forming a sharp concentration boundary within this cell. In $\mathbf{f}-\mathbf{h}$, only PEAR and HD-ZIP concentrations are shown, whilst all model components are shown here in all cases. e, Quantification of expression level of CNA-GFP and PEAR1-GFP at 4-cell region in wild-type background. $n$ represents individual measurements across 3 (CNA-GFP) or 4 (PEAR1-GFP) independent biological samples, respectively. Bar graphs represent mean. Error bars are s.d. Dots, individual data points. f, Including all three interactions labelled in panel $\mathbf{c}$ in the model, results in the formation of sharp concentration gradients of PEAR1 (black line) and HD-ZIP III (blue line) with the boundary forming in the PSE-IN. g, In simulations where HD-ZIP III does not regulate PEAR1 diffusion (Interaction (3) in panel c), PEAR1 protein is predicted to spread into the procambium and metaxylem as shown in Fig. 4k-m and Extended Data Fig. 4. h, In simulations where PEAR1 does not activate HD-ZIP III transcription (Interaction (1) in panel c), the concentration of HD-ZIP III is reduced in the PSE-IN cell as shown in Fig. 4h-j. i, A regulatory mechanism forming the boundary between a dividing and a non-dividing cell during procambial development. White, orange, dark-green and light-green arrowheads indicate PSE, PSE-LN, PSE-IN and IPC. Scale bars, $25 \mu \mathrm{m}$. 


\section{Supplementary Table legends}

\section{Supplementary Table 1|}

This file contains a list of primers and plasmids used in this study.

\section{Supplementary Table 2}

This file contains a list of putative PEAR1/PEAR2 direct targets and their description.

\section{Supplementary Table 3|}

This file contains individual $P$-values for Tukey's HSD test (for Fig. $2 \mathrm{f}$ and Extended Data Fig. 5b-d) and for two-sided Student's t-test (for Extended Data Fig. 7b). For Tukey's HSD test, data contains: difference in mean values (diff), the lower end point of the interval (lwr), the upper end point of the interval (upr) and $p$-value after adjustment for the multiple comparisons ( $\mathrm{p}$ adj). 


\section{SUPPLEMENTARY INFORMATION}

\section{Supplementary Notes}

\subsection{Mapping of the position of periclinal cell divisions}

As previously reported, the cell identity in phloem/procambium is not fully correlated with the cell lineage. For example, the cell at the protophloem sieve element (PSE) position undergoes two types of periclinal cell divisions, in which PSE initially generates procambium cell files inward, and the later produces metaphloem sieve element (MSE) (Extended Data Fig. 1a). In addition, companion cells (CC) are produced by the divisions in procambial cells laterally adjacent to PSE (Extended Data Fig. 1a). In order to categorize each periclinal cell division based on the position of cells, we therefore classified the phloem/procambium cells into five groups based on their position relative to PSE and pericycle (Fig. 1a). The cells surrounding the PSE were classified into two group, PSE-lateral neighbour (PSE-LN), a cell touching both PSE and pericycle, and PSE- internal neighbour (PSE-IN), a cell touching PSE but not pericycle (Fig. 1a). The intervening procambial cells non-adjacent to PSE were classified into two categories, outer procambial cell (OPC), a cell adjacent to pericycle, and internal procambial cell (IPC), a cell touching neither PSE nor pericycle. By comparing the cell pattern in segmented images (Extended Data Fig. 1b), 273 periclinal cell divisions from 13 independent wild-type roots were mapped, resulting in 60 events in PSE, 142 events in PSE-LN, 39 events in OPC, 6 in PSE-IN and 26 events in PX position, respectively (Extended Data Fig. 1c). The number of cells in each position is different, for example, two in PSE and four in PSE-LN. Also the number of OPC or IPC increases after the periclinal division in PSE or PSE-LN during development, and therefore we counted the number of cells in each cell category in each cross-section and calculated the mean number of cells in a given cell type (Extended Data Fig. 1d). The number of events per cell in each group was calculated by diving the number of events by the mean cell number of each group during development (Fig. 1b and Extended Data Fig.1).

\subsection{Redundancy of PEAR genes}

In Arabidopsis, the family of DOF-domain transcription factors comprises 36 members (Extended Data Fig. 3b). Analysis of pearl, its closest homolog pear2 or the double pear1pear2 mutants did not reveal phenotypes similar to the pPEAR1[XVE]::icals3m line 
affected in symplastic movement between phloem and procambium cells (Extended Data Fig 2a-e), suggesting broader functional redundancy within the family of DOF TFs. In order to understand the range of this effect we generated multiple combinatorial mutants covering multiple phloem specific/abundant members of the DOF family.

As a first approach we generated combinatorial mutants with the close homologs of PEARl (Extended Data Fig. 3b). Knocking-out up to five genes from the PEARl clade (pear1 pear2 obp2 obp3 dof2.2 (At2g28810)), did not result in a phenotype resembling the icals3m line (Extended Data Fig. 5b). At the same time, screening of the transcriptional reporters of DOF family members identified DOF5.6/HCA2, DOF3.2/DOF6 and DOF5.3/TMO6 as genes expressed specifically/abundantly in the early phloem position (Extended Data Fig. 3d). We measured expression levels of these genes in the pearl pear 2 mutant background and found that transcript levels of $H C A 2$, DOF6 and TMO6 were elevated (Extended Data Fig. 3e), suggesting a compensation mechanism among more distantly related PEAR genes. In this scenario, the phenotype of the double mutant might be obscured by the increased expression level of other PEAR genes and only absence of all of them would result in strong phenotypes.

To test this hypothesis we generated multiple combinatorial pear mutants using available KO lines for PEAR1, PEAR2, DOF6, OBP2 and HCA2. The intermediate triple and all quadruple mutant combinations did not show a narrow root phenotype (Extended Data Fig. 5b), however, when combining five mutations together we found that around 30 per cent of the quintuple pear1 pear2 obp2 dof6 hca2 mutants displayed reduced root growth. These roots showed a reduction in the number of the procambial cell files, and about 30 per cent of them resembled the phenotype of roots impaired in the symplastic communication around PSE (pPEAR1[XVE]::icals3m, Extended Data Fig.2c). In those roots some of the procambial cell files did not undergo any periclinal divisions. Specifically comparison of all the intermediate quadruple mutants with a quintuple mutant indicates the importance of each individual gene in the regulation of phloem and procambium proliferation. Expression of PEAR1, PEAR2, OBP2 and HCA2 genes under their native promoters significantly increased the number of vascular cell files suppressing the strong phenotype of the quintuple mutant. The analysed lines expressing DOF6 showed a relatively weak phenotype suppression effect in the quintuple mutant background.

Since the penetrance of the strong phenotype in the quintuple pearl pear 2 obp 2 dof6 hca 2 mutants was not very high, we introduced a mutation in TMO6 gene, a closest homolog of DOF6 expressed abundantly in phloem and upregulated in the pear1 pear2 double mutant background (Extended Data Fig. 3e). After introducing CRISPR-Cas 9 
generated loss-of-function allele of TMO6 in pear quintuple (resulting in the pear1 pear2 dof6 tmo6 obp2 hca2 hextuple mutant), even less cell divisions occurred in the root vasculature, reducing the variability between roots. The number of procambial cell files in the differentiated zone of the pear hextuple mutant $(\sim 10)$ closely corresponds to the number of procambial initials $(\sim 7)$ suggesting that almost all periclinal cell divisions are abolished in this mutant background. Importantly, we did not observe any cell division phenotype in the single tmo6 mutant (Extended Data Fig. 5b), suggesting that TMO6 functions redundantly with the other PEAR genes. To further support the importance of $P E A R$ genes in the process of periclinal division of the procambial cells, we expressed them individually under their native promoters in the hextuple mutant background. PEAR1, DOF6 and TMO6 suppressed the strong phenotype of pear hextuple mutant confirming the role of PEAR genes in regulation of this process (Extended Data Fig.5d). Expression analysis of the pPEAR2::PEAR2-VENUS in the pear hextuple mutant revealed lack of $P E A R 2$ promoter activity in the early phloem. Since most of the periclinal divisions are concentrated around the early phloem cells, pPEAR2::PEAR2-VENUS did not suppress this phenotype in the pear hextuple mutant. By contrast, suppression was very clear in pear quintuple where PEAR2 promoter is active in the early phloem cells (Extended Data Fig. 5d).

Because of the strong effect of tmo6 mutation in the hextuple mutant background, we investigated its influence on the pearl, pear2 and dof6 mutants. Double mutants pear 1 tmo6, pear2 tmo6 and dof6 tmo6 did not show the strong phenotype observed in the quintuple or hextuple mutants. The triple mutant pearl pear2 tmo6 showed a strong phenotype but not to the extent of the hextuple mutant (Fig 2f). This phenotype was also strongly variable suggesting that although TMO6 plays an important role, the contribution from other pear mutants is required for a strong hextuple mutant phenotype. Furthermore, we have established the phenotypes for pear 1 pear 2 dof6 tmo 6 quadruple and pear 1 pear 2 dof6 tmo6 hca2 quintuple mutant (Fig. 2f and Extended Data Fig. 5b). These mutants largely resemble the pearl pear2 dof6 tmo6 hca2 obp2 hextuple mutant, indicating that the mobile PEAR1, PEAR2, DOF6 and TMO6 proteins play a major role in regulating radial growth, while $\mathrm{HCA} 2$ and $\mathrm{OBP} 2$ play a more minor role.

\subsection{Uncoupling the cell division and cell differentiation effects of the pear mutants}

In addition to the reduction of vascular cell number, we made observations of cells that had not cleared the cytoplasm (characteristic to sieve element differentiation) in some of the pear combinatorial mutants. To assess the status of phloem, we were assaying phloem transport and unloading using the CFDA dye (Oparka et al., 1994). We found strong 
transport defects in the pear hextuple mutant, indicating problems in the functionality of phloem. In contrast, we observed functional CFDA transport and unloading in the narrow roots of pearl pear2 dof6 obp2 hca2 quintuple mutant (Extended Data Fig. 5e). Thus, the problems in the PEAR mediated periclinal cell divisions can be dissected from the apparent defects in phloem differentiation. Furthermore, to exclude possibility that defects observed in phloem function and differentiation in the pear hextuple mutant influence the rate of periclinal divisions, we counted the number of vascular cell files at an early stage of plant development, before phloem transport becomes active. Previous work has showed that the activation of phloem transport occurs only around two days after germination, when phloem becomes fully functional as a consequence of PSE enucleation (Bauby et al. 2007) and the procambium/phloem tissue proliferation stage precedes developmentally the final differentiation of PSE (Furuta et al., 2014). We observed reduced number of vascular cell files in the postembryonic root of 1.5 days old hextuple mutant seedlings (Extended Data Fig. 5f), thus dissecting the cell proliferation and differentiation aspects of the pear hextuple mutant phenotype.

\subsection{Bisymmetric auxin-cytokinin response pattern in root}

Previous work indicated that auxin-induced cytokinin production in the xylem axis triggers the periclinal cell divisions in a non-cell autonomous manner in the flanking phloem/procambial domain (De Rybel et al., 2014). As described in Fig. 1, the periclinal cell divisions are concentrated around the PSE, whereas no periclinal cell division was observed in those internal procambial cells (IPC, Fig. 1a and b). Here we further dissected the dynamics of the hormonal response domain during procambial development. Auxin signalling maximum was formed in xylem cells already at initial stage (Extended Data Fig. 7a') and maintained during procambial development (Extended Data Fig. 7a" and a"'). By contrast, we found that the domain of high cytokinin response is more dynamic. At initial stage, high cytokinin response was activated at PSE and its neighbouring cells (Extended Data Fig. 7a'), and this high cyokinin response domain was maintained during proliferative phase (Extended Data Fig. 7a'-a"). Only at a later stage of development, we observed that cytokinin response domain becomes concentrated in procambial cells neighbouring the xylem axis (Extended Data Fig. 7a"').

\subsection{Interaction of PEAR1 and cytokinin signalling during embryogenesis}

As described in main text, we revealed the interaction of PEAR1 and cytokinin sigalling in post-embryonic root vascular tissue (Fig. 3a-e). We further studied this interaction and its dynamics during embryogenesis where the root vascular cells are initiated. During 
embryogenesis, high cytokinin response is initiated in vascular cells of upper lower tier (ult) at the early heart stage (Extended Data Fig. 7e and f), and only at the late heart stage is the characteristic bisymmetric pattern of cytokinin output established (Extended Data Fig. $7 \mathrm{~g}$ and $\mathrm{h}$ ). In wol embryos, activation of cytokinin response in vascular tissue does not occur and a radial auxin response pattern is maintained (Extended Data Fig. 7j-1). PEAR1 transcription pattern was highly correlated with cytokinin signalling during embryogenesis, except for its broad expression in the early globular stage (Extended Data Fig. 7m-o). By contrast, in wol embryos, where no cytokinin response was detected within vascular cells, PEARl transcription was initially observed in the globular wol embryo (Extended Data Fig. 7p) but was gradually attenuated after heart stage (Extended Data Fig.7q-r).

\subsection{Analysis of PEAR1/2 downstream targets}

Studying the expression patterns of PEAR1/2 downstream targets by in silico analysis and reporter constructs revealed that most of PEAR1/2 targets are expressed in PSE and its surrounding cells, indicating that PEAR genes control their targets in a non-cell autonomous manner. This result highlights that the mobility of the PEAR1/2 proteins is important for their function (Fig. 2g-h and Extended Data Fig.6a-i). In order to dissect the function of PEAR1/2 targets, we performed a statistical overrepresentation test for Gene Ontology (GO) terms of the PEAR1/2 direct targets using the PANTHER (protein annotation through evolutionary relationship) classification system (http://www.pantherdb.org/). However, no statistically significant results were found. In addition, we could not find genes previously shown to regulate cell proliferation (Supplementary Table 2), suggesting that PEAR1/PEAR2 control radial growth through still uncharacterized genes. By overexpressing some of the targets we found SMXL3 to be able to induce periclinal cell division (Extended Data Fig.6j).

\section{Supplemental references}

Oparka, K. J., Duckett, C. M., Prior, D. A. M. \& Fisher, D. B. Real - time imaging of phloem unloading in the root tip of Arabidopsis. The Plant Journal 6, 759-766, doi:doi:10.1046/j.1365-313X.1994.6050759.x (1994).

De Rybel, B. et al. Plant development. Integration of growth and patterning during 
vascular tissue formation in Arabidopsis. Science 345, 1255215, doi:10.1126/science.1255215 (2014).

Bishopp, A. et al. A mutually inhibitory interaction between auxin and cytokinin specifies vascular pattern in roots. Curr Biol 21, 917-926, doi:10.1016/j.cub.2011.04.017 (2011).

Bauby, H., Divol, F., Truernit, E., Grandjean, O. \& Palauqui, J. C. Protophloem differentiation in early Arabidopsis thaliana development. Plant Cell Physiol 48, 97-109, doi:10.1093/pcp/pc1045 (2007).

Furuta, K. M. et al. Plant development. Arabidopsis NAC45/86 direct sieve element morphogenesis culminating in enucleation. Science 345, 933-937, doi:10.1126/science.1253736 (2014). 


\section{Supplementary Modelling Information}

\section{Model aims and philosophy}

To date there have been several mechanisms shown to be capable of generating either gradients or sharp domains of differential gene expression. Although some aspects of the interaction between PEAR proteins and HD-ZIP III's are similar to other patterning mechanisms based on transcriptional regulation, the observation that $\mathrm{HD}$-ZIP III has a dual effect on both PEAR transcription and PEAR mobility appeared to be a new type of interaction that had not been explored before. Therefore, through this modelling approach, we explore the effect that these processes have on regulating the spatial domains of both PEAR and HD-ZIP III within the procambium during phloem specification.

In order to model a single boundary between domains of PEAR and HD-ZIP III, we consider only a single generic PEAR gene and a single generic HD-ZIP III gene rather than modelling individual family members. As this patterning process occurs in a single dimension (i.e. from the centre of the root to the margin) we chose to model this as a multi-compartment model in one dimension, with compartments representing either cell wall or sub-cellular space. In addition to PEAR and HD-ZIP III, the model also considers auxin, cytokinin and miRNA165/6 as these are all components that have been shown to regulate either PEAR or HD-ZIP III (Donner et al. 2009; Carlsbecker et al. 2010; Miyashima et al. 2011). In this model auxin and miRNA165/6 can be considered as inputs to the model, with cytokinin distribution leading from the auxin distribution, while predictions for PEAR and HD-ZIP III spatial distribution can be considered as the key outputs originating from this study. As there have been a suite of models predicting the localisation of auxin, cytokinin and miRNA165/6 during root vascular patterning (Muraro et al. 2014; De Rybel et al. 2014; el Showk et al. 2015; Mellor et al. 2016), we do not seek to reproduce these findings and instead impose the spatial patterning of these three components as has been described in previous models. As formulated, the model only requires spatial positioning of an auxin source at one end of the template (in the metaxylem) and a miRNA source at the other end of the template (at the outer edge of the stele) in order to produce the spatial distribution of cytokinin, HD-ZIP III and PEAR.

\subsection{Inputs: Auxin and miRNA}

In this model we impose an auxin maximum in the xylem axis, in a similar way to that shown in mathematical models of root vascular patterning and supported by experimental data of response markers. Rather than incorporating auxin transporters into our model, we limit auxin production to the metaxylem and set a low diffusion parameter relative to auxin degradation so that the vast majority of auxin remains in the metaxylem, but that a low level exists in adjacent cells. The final auxin distribution is similar to previous models (Muraro et al. 2014; De Rybel et al. 2014; el Showk et al. 2015).

The effect of miRNA on the spatial distribution of the HD-ZIP III transcription factor PHB is modulated by the interaction and mutual degradation with a miRNA and has been explored mathematically in Muraro et al. (2014). In this model, the miRNA was produced in response to $S H R$ in the endodermis and could then diffuse into the stele.

Here, we simplify this by assuming a constant source of miRNA at the outer edge of the stele. The miRNA can then diffuse within the stele and degrade (and be degraded by) HD-ZIP III. 


\subsection{Outputs: Cytokinin, PEAR and HD-ZIP}

Although we do not explicitly set out to predict cytokinin distribution, it emerges from our model. Cytokinin biosynthesis is promoted by auxin response in the xylem axis via MP-dependent activation of $L O G$ genes (De Rybel et al. 2014) whilst its activity is repressed in these cells via AHP6 (Mahonen et al. 2006). For this reason we include separate model components representing both the hormone itself (assumed to be produced directly in response to auxin) and a generic cytokinin response gene (assumed to be repressed by auxin directly). It is this cytokinin response that then promotes PEAR production. In this way, while PEAR production may occur wherever the cytokinin response gene is present, it is effectively excluded from the metaxylem due to the auxin source there. As has been done for previous models, we make the assumption that while the cytokinin hormone is free to move via diffusion, the cytokinin response is not.

As we show here that $P E A R$ transcription is promoted by cytokinin, we use the cytokinin response (described above) to promote PEAR production in the model. Conversely, we show that HD-ZIP III represses PEAR transcription, so the PEAR production rate in the model is negatively affected by the level of HD-ZIP III. Based on the observed movement of translational PEAR reporters, we assume that PEAR can move via diffusion, but that this rate of movement is negatively affected by the presence of HD-ZIP III as supported by experimental determination of the diffusion coefficients.

Though we use a single generic HD-ZIP III protein we assume three independent modules contribute to its production. Firstly we assume a basal constant production rate in all cells, production is then increased in response to auxin and decreased in response to PEAR. Although there are differences in the regulation of individual HD-ZIP III's, e.g. ATHB8 shows a clear auxin induction, we feel that, collectively, these rules reflect the activity of the group. In addition to a miRNA independent rate of degradation (as is included for all model components), we also incorporate a mechanism through which HD-ZIP III and miRNA mutually degrade one another as described above.

Based on these regulatory interactions, we can predict the steady state patterns of cytokinin, HDZIP III and PEAR based on two inputs namely auxin and miRNA165/6. This allows us to dissect the network and examine the effects that each regulatory component has on the final pattern.

\section{Model Description}

\subsection{Spatial Domain}

The model is solved on a one-dimensional spatial array of discrete compartments representing a crosssection of root tissue from the centre of the stele at the xylem axis to the edge of the stele where phloem is formed. The spatial subdivisions may represent either cell or cell wall compartments, with multiple compartments per cell so that intracellular resolution is present within the model. Either 3,4 or 5 cells are simulated, subdivided by 2,3 or 4 walls respectively. All compartments have equal unit widths, with 23 compartments per cell and 2 compartments per cell wall. Each compartment is numbered sequentially from 1 (xylem) to $N$ (outer stele) where $N$ is the total number of compartments. The subset of cellular compartments is denoted $K$ and the subset of wall components denoted $W$.

\subsection{Model Components}

The model simulates the evolution over time in a given spatial compartment $i$ of a miRNA (denoted $M_{i}$ ), a generic HD-ZIP III (denoted $H_{i}$ ), PEAR (denoted $P_{i}$ ), auxin (denoted $A_{i}$ ), cytokinin (denoted $C_{i}$ ) and the cytokinin response (denoted $R_{i}$ ). Differentiating cytokinin from the cytokinin response in this way is necessary to capture the dual role of auxin in promoting cytokinin biosynthesis while also repressing the cytokinin response (De Rybel et al. 2014).

The spatial movement of model components via diffusion is modelled using standard discretisations of diffusion operators to simulate flux between adjacent compartments, with the exception of the movement of PEAR. In the case of PEAR to simulate the blocking of PEAR movement by HD-ZIP 
III the diffusion coefficient between two adjacent compartments is modified by a decreasing function of the average value of HD-ZIP III in the two compartments:

$$
J=D_{p}\left(\frac{P_{i-1}-P_{i}}{1+\left(\frac{H_{i-1}+H_{i}}{2 \phi}\right)^{q}}\right)
$$

where $J$ is the flux of PEAR between compartments $i$ and $i-1, D_{p}$ is the diffusion coefficient in the absence of HD-ZIP III and $\phi$ and $m$ are additional parameters. For values and definition of these and all other parameters please refer to Table 1.

For simplicity, when modelling the three genes HD-ZIP III, PEAR and the generic cytokinin response we use a single variable to represent both the mRNA and protein. Gene expression is modelled using combinations of Hill functions, depending on the required regulatory logic. We assume $H D-Z I P$ $I I I$ is activated by three independent modules, one constitutive, one auxin dependent and one PEAR dependent, and so model the transcription rate of $H D-Z I P I I I F_{h}$ as the sum of a constant, and increasing functions of auxin and PEAR:

$$
F_{h}(A, P)=\lambda\left(1+\frac{A^{n_{h}}}{\theta_{h}^{n_{h}}+A^{n_{h}}}+\frac{P^{m_{h}}}{\psi_{h}^{m_{h}}+P^{m_{h}}}\right),
$$

where $\theta_{h}$ and $\psi_{h}$ are threshold parameters, $n_{h}$ and $m_{h}$ Hill coefficents, and $\lambda$ a proportionality constant.

PEAR is activated by the cytokinin response and repressed by HD-ZIP III so we model its transcription $F_{p}$ as the product of positive and negative Hill functions:

$$
F_{p}(H, R)=\frac{\theta_{p}^{n_{p}}}{\theta_{p}^{n_{p}}+H^{n_{p}}} \times \frac{R^{m_{p}}}{\psi_{p}^{m_{p}}+R^{m_{p}}},
$$

where $\theta_{p}$ and $\psi_{p}$ are threshold parameters and $n_{p}$ and $m_{p}$ Hill coefficents.

Cytokinin production $F_{c}$ is modelled as a simple increasing Hill function of auxin:

$$
F_{c}(A)=\frac{A^{n_{c}}}{\theta_{c}^{n_{c}}+A^{n_{c}}}
$$

where $\theta_{c}$ is the threshold parameter and $n_{c}$ the Hill coefficent.

Finally the cytokinin response is regulated positively by cytokinin, but negatively by auxin so its transcription rate $F_{r}$ is given by:

$$
F_{r}(A, C)=\frac{\theta_{r}^{n_{r}}}{\theta_{r}^{n_{r}}+A^{n_{r}}} \times \frac{C^{m_{r}}}{\psi_{r}^{m_{r}}+C^{m_{r}}}
$$

where $\theta_{r}$ and $\psi_{r}$ are threshold parameters and $n_{r}$ and $m_{r}$ Hill coefficents.

For all of these production rate functions we make the approximation that the rate of production is equal throughout a given cell. This is done by using the mean value of any given transcription factor within the set of compartments making up that cell in the above functions. We denote these mean cellular values for a given compartment using the ${ }^{-}$notation so that for example the rate of cytokinin production in a compartment $i$ is a function of $\widehat{A}_{i}$, the mean value of auxin in all compartments in the cell containing $i$.

The modelling of the mutual degradation of miRNA and HD-ZIP III is simulated via mass-action and is similar to that of Muraro et al. (2014). Degradation terms for each model component are included in all cellular compartments. 


\subsection{Cellular compartments}

Combining the above for $1<i<N$ and $i \in K$ (cellular compartments away from the boundary) we have the following set of ordinary differential equations:

$$
\begin{aligned}
\frac{d M_{i}}{d t} & =D_{m}\left(M_{i-1}+M_{i+1}-2 M_{i}\right)-\mu_{m} M_{i}-\eta_{m} M_{i} H_{i} \\
\frac{d H_{i}}{d t} & =\mu_{h}\left(F_{h}\left(\widehat{A}_{i}, \widehat{P}_{i}\right)-H_{i}-\eta_{h} M_{i} H_{i}\right) \\
\frac{d P_{i}}{d t} & =\mu_{p}\left(F_{p}\left(\widehat{H}_{i}, \widehat{R}_{i}\right)-P_{i}\right)+D_{p}\left(\frac{P_{i-1}-P_{i}}{1+\left(\frac{H_{i-1}+H_{i}}{2 \phi}\right)^{q}}+\frac{P_{i+1}-P_{i}}{1+\left(\frac{H_{i}+H_{i+1}}{2 \phi}\right)^{q}}\right), \\
\frac{d A_{i}}{d t} & =\alpha_{i}-A_{i}+D_{a}\left(A_{i-1}+A_{i+1}-2 A_{i}\right), \\
\frac{d C_{i}}{d t} & =\mu_{c}\left(F_{c}\left(\widehat{A}_{i}\right)-C_{i}\right)+D_{c}\left(C_{i-1}+C_{i+1}-2 C_{i}\right), \\
\frac{d R_{i}}{d t} & =\mu_{r}\left(F_{r}\left(\widehat{A}_{i}, \widehat{C}_{i}\right)-R_{i}\right)
\end{aligned}
$$

where $D_{m}, D_{a}$ and $D_{c}$ are the respective diffusion coefficients of miRNA, auxin and cytokinin, $\mu_{m}$, $\mu_{h}, \mu_{p}, \mu_{c}$ and $\mu_{r}$ are turnover rates of miRNA, HD-ZIP III, PEAR and cytokinin and the cytokinin response and $\eta_{m}$ and $\eta_{h}$ are the mutual degradation rates of miRNA and HD-ZIP III. $\alpha_{i}$ is the production rate of auxin and is set to be zero except for the in compartments in the first cell representing the xylem axis.

\subsection{Wall compartments}

For the wall compartments $(i \in W)$ we set production and degradation equal to zero and only model movement and the mutual degradation of HD-ZIP III and miRNA so that:

$$
\begin{aligned}
\frac{d M_{i}}{d t} & =D_{m}\left(M_{i-1}+M_{i+1}-2 M_{i}\right)-\eta_{m} M_{i} H_{i}, \\
\frac{d H_{i}}{d t} & =0, \\
\frac{d P_{i}}{d t} & =D_{p}\left(\frac{P_{i-1}-P_{i}}{1+\left(\frac{H_{i-1}+H_{i}}{2 \phi}\right)^{q}}+\frac{P_{i+1}-P_{i}}{1+\left(\frac{H_{i}+H_{i+1}}{2 \phi}\right)^{q}}\right), \\
\frac{d A_{i}}{d t} & =D_{a}\left(A_{i-1}+A_{i+1}-2 A_{i}\right), \\
\frac{d C_{i}}{d t} & =D_{c}\left(C_{i-1}+C_{i+1}-2 C_{i}\right), \\
\frac{d R_{i}}{d t} & =0 .
\end{aligned}
$$




\subsection{Boundary compartments}

At the boundary representing the centre of the stele $(i=1)$ we have zero flux boundary conditions so that:

$$
\begin{aligned}
\frac{d M_{1}}{d t} & =D_{m}\left(M_{2}-M_{1}\right)-\mu_{m} M_{1}-\eta_{m} M_{1} H_{1} \\
\frac{d H_{1}}{d t} & =\mu_{h}\left(F_{h}\left(\widehat{A}_{1}, \widehat{P}_{1}\right)-H_{1}-\eta_{h} M_{1} H_{1}\right) \\
\frac{d P_{1}}{d t} & =\mu_{p}\left(F_{p}\left(\widehat{H}_{1}, \widehat{R}_{1}\right)-P_{1}\right)+D_{p}\left(\frac{P_{2}-P_{1}}{1+\left(\frac{H_{1}+H_{2}}{2 \phi}\right)^{q}}\right), \\
\frac{d A_{1}}{d t} & =\alpha_{1}-A_{1}+D_{a}\left(A_{2}-A_{1}\right), \\
\frac{d C_{1}}{d t} & =\mu_{c}\left(F_{c}\left(\widehat{A}_{1}\right)-C_{1}\right)+D_{c}\left(C_{2}-C_{1}\right) \\
\frac{d R_{1}}{d t} & =\mu_{r}\left(F_{r}\left(\widehat{A}_{1}, \widehat{C}_{1}\right)-R_{1}\right)
\end{aligned}
$$

and finally at the outer stele boundary $(i=N)$ we also have zero flux boundary conditions, except for miRNA which is held fixed at $M_{b n d}$ so that:

$$
\begin{aligned}
\frac{d M_{N}}{d t} & =D_{m}\left(M_{N-1}-2 M_{N}+M_{b n d}\right)-\mu_{m} M_{N}-\eta_{m} M_{N} H_{N} \\
\frac{d H_{N}}{d t} & =\mu_{h}\left(F_{h}\left(\widehat{A}_{N}, \widehat{P}_{N}\right)-H_{N}-\eta_{h} M_{N} H_{N}\right) \\
\frac{d P_{N}}{d t} & =\mu_{p}\left(F_{p}\left(\widehat{H}_{N}, \widehat{R}_{N}\right)-P_{N}\right)+D_{p}\left(\frac{P_{N-1}-P_{N}}{1+\left(\frac{H_{N-1}+H_{N}}{2 \phi}\right)^{q}}\right) \\
\frac{d A_{N}}{d t} & =-A_{N}+D_{a}\left(A_{N-1}-A_{N}\right) \\
\frac{d C_{N}}{d t} & =\mu_{c}\left(F_{c}\left(\widehat{A}_{N}\right)-C_{N}\right)+D_{c}\left(C_{N-1}-C_{N}\right) \\
\frac{d R_{N}}{d t} & =\mu_{r}\left(F_{r}\left(\widehat{A}_{N}, \widehat{C}_{N}\right)-R_{N}\right)
\end{aligned}
$$

\subsection{Model Parameters}

For model parameter values see Table 1. All parameter values are estimates within reasonable bounds based on trial and error in order to demonstate the plausibilty of the model in reproducing experimental observations. Since the model is dimensionless, in the absence of spatial effects, variables are mostly constrained between 0 and 1 , and the parameter values are also dimensionless. In particular the Hill thresholds and coefficients represent the relative sensitivity of the different regulatory mechanisms present, with most set to default values of 0.1 and 1 respectively. The relative values of the parameters relating to the mutual degradation of miRNA and HD-ZIP III are similar to those used in Muraro et al. (2014), while values for the turnover rates of model variables have little or no effect on model steady state. Selecting appropriate values for the relative diffusion coefficents is essential in order to observe gradients in the model components over the desired spatial scales, in the experimentally observed positions. While using other parameter values is likely to alter both the position and magnitude of these gradients, we find that small perturbations from the selected parameter set does not significantly affect the overall patterns produced by the model. 
Table 1: Nondimensional parameters, with default values.

\begin{tabular}{|l|l|}
\hline Diffusion coefficients & \\
\hline \hline$D_{m}$ & 400 \\
$D_{p}$ & 300 \\
$D_{a}$ & 10 \\
$D_{c}$ & 1000 \\
$\phi$ & 0.005 \\
$q$ & 2 \\
\hline \hline Transcription parameters & \\
\hline \hline$\lambda$ & 0.5 \\
$\theta_{h}$ & 1 \\
$\psi_{h}$ & 1 \\
$n_{h}$ & 1 \\
$m_{h}$ & 1 \\
$\theta_{p}$ & 0.2 \\
$\psi_{p}$ & 0.1 \\
$n_{p}$ & 4 \\
$m_{p}$ & 1 \\
$\theta_{c}$ & 0.1 \\
$n_{c}$ & 1 \\
$\theta_{r}$ & 0.1 \\
$\psi_{r}$ & 0.1 \\
$n_{r}$ & 1 \\
$m_{r}$ & 1 \\
\hline \hline Turnover rates & \\
\hline \hline$\mu_{h}$ & 1 \\
$\mu_{p}$ & 1 \\
$\mu_{c}$ & 1 \\
$\mu_{r}$ & 1 \\
\hline \hline miRNA / HD-ZIP III interaction & \\
\hline \hline$\eta_{m}$ & 500 \\
$\eta_{h}$ & 1000 \\
$M_{b n d}$ & 1 \\
$\mu_{m}$ & 1 \\
\hline \hline Auxin production & \\
\hline \hline$\alpha_{i}$ (i in xylem cell) & 1 \\
$\alpha_{i}$ (i not in xylem cell) & 0 \\
\hline & \\
\hline
\end{tabular}




\section{Alternate Cases}

\subsection{No block on PEAR movement by HD-ZIP III}

For the case where the block on PEAR movement in the presence of HD-ZIP III is removed, we simply replace the HD-ZIP III modified diffusion operator given by Equation (1) used in Equations (6c), (7c), $(8 \mathrm{c})$ and $(9 \mathrm{c})$ with the standard discretised diffusion operator so that in general:

$$
\frac{d P_{i}}{d t}=\mu_{p}\left(F_{p}\left(\widehat{H}_{i}, \widehat{R}_{i}\right)-P_{i}\right)+D_{p}\left(P_{i-1}-2 P_{i}+P_{i+1}\right),
$$

with zero flux boundary conditions defined as before.

\subsection{No positive feedback on HD-ZIP III expression from PEAR}

To omit the positive feedback from PEAR on HD-ZIP III we replace Equation (2) with:

$$
F_{h}(A)=\lambda\left(1+\frac{A^{n_{h}}}{\theta_{h}^{n_{h}}+A^{n_{h}}}\right)
$$

so that HD-ZIP III production is only dependent on auxin plus a constitutive component.

\subsection{No negative feedback on PEAR expression from HD-ZIP III}

To omit the negative feedback from HD-ZIP III on PEAR we replace Equation (3) with:

$$
F_{p}(R)=\frac{R^{m_{p}}}{\psi_{p}^{m_{p}}+R^{m_{p}}}
$$

so that PEAR production is only dependent on the cytokinin response.

\section{Model Implementation}

The model is implemented using the Python 2.7 programming language with the 'odeint' function from the Scipy package used to solve the differential equations (Jones et al. 2001-) and Matplotlib (Hunter 2007) used to plot the state of the model after 100,000 timesteps, which we assume to be at steady state.

\section{References}

Annelie Carlsbecker, Ji-Young Lee, Christina J. Roberts, Jan Dettmer, Satu Lehesranta, Jing Zhou, Ove Lindgren, Miguel A. Moreno-Risueno, Anne Vatén, Siripong Thitamadee, and et al. Cell signalling by microRNA165/6 directs gene dose-dependent root cell fate. Nature, 465(7296):316321, Apr 2010.

Bert De Rybel, Milad Adibi, Alice S. Breda, Jos R. Wendrich, Margot E. Smit, Ondřej Novák, Nobutoshi Yamaguchi, Saiko Yoshida, Gert Van Isterdael, Joakim Palovaara, Bart Nijsse, Mark V. Boekschoten, Guido Hooiveld, Tom Beeckman, Doris Wagner, Karin Ljung, Christian Fleck, and Dolf Weijers. Plant development. integration of growth and patterning during vascular tissue formation in Arabidopsis. Science, 345(6197):1255215, Aug 2014.

Tyler J. Donner, Ira Sherr, and Enrico Scarpella. Regulation of preprocambial cell state acquisition by auxin signaling in Arabidopsis leaves. Development, 136(19):3235-3246, 2009. 
Sedeer el Showk, Hanna Help-Rinta-Rahko, Tiina Blomster, Riccardo Siligato, Athanasius F. M. Marée, Ari Pekka Mähönen, and Verônica A. Grieneisen. Parsimonious model of vascular patterning links transverse hormone fluxes to lateral root initiation: Auxin leads the way, while cytokinin levels out. PLOS Computational Biology, 11(10):e1004450, Oct 2015.

J. D. Hunter. Matplotlib: A 2d graphics environment. Computing In Science Ė Engineering, 9(3): 90-95, 2007.

Eric Jones, Travis Oliphant, Pearu Peterson, et al. SciPy: Open source scientific tools for Python, 2001-. URL http://www.scipy.org/.

A. P. Mahonen, Bishopp A., Higuchi M., Nieminen K.M., Kinoshita K., Törmakängas K., Ikeda Y., Oka A., Kakimoto T., and Helariutta Y. Cytokinin signaling and its inhibitor AHP6 regulate cell fate during vascular development. Science, 311(5757):94-98, Jan 2006.

Nathan Mellor, Milad Adibi, Sedeer El-Showk, Bert De Rybel, John King, Ari Pekka Mähönen, Dolf Weijers, and Anthony Bishopp. Theoretical approaches to understanding root vascular patterning: a consensus between recent models. Journal of Experimental Botany, 68(1):5-16, Nov 2016.

Shunsuke Miyashima, Satoshi Koi, Takashi Hashimoto, and Keiji Nakajima. Non-cell-autonomous microRNA165 acts in a dose-dependent manner to regulate multiple differentiation status in the Arabidopsis root. Development, 138(11):2303-2313, 2011.

Daniele Muraro, Nathan Mellor, Michael P. Pound, Hanna Help, Mikaël Lucas, Jérôme Chopard, Helen M. Byrne, Christophe Godin, T Charlie Hodgman, John R. King, Tony P. Pridmore, Ykä Helariutta, Malcolm J. Bennett, and Anthony Bishopp. Integration of hormonal signaling networks and mobile microRNAs is required for vascular patterning in Arabidopsis roots. Proc Natl Acad Sci U S A, 111(2):857-862, Jan 2014. 